\title{
Nigrostriatal overabundance of $\alpha$-synuclein leads to decreased vesicle density and deficits in dopamine release that correlate with reduced motor activity
}

\author{
Meret Nora Gaugler • Ozgur Genc - Wojciech Bobela - Safa Mohanna - Mustafa Taleb Ardah • \\ Omar Mukhtar El-Agnaf • Marco Cantoni · Jean-Charles Bensadoun • Ralf Schneggenburger • \\ Graham W. Knott • Patrick Aebischer • Bernard Laurent Schneider
}

Received: 19 August 2011/Accepted: 14 February 2012/Published online: 24 February 2012

(C) Springer-Verlag 2012

\begin{abstract}
Synuclein ( $\alpha$-syn) is a presynaptic protein present at most nerve terminals, but its function remains largely unknown. The familial forms of Parkinson's disease associated with multiplications of the $\alpha$-syn gene locus indicate that overabundance of this protein might have a detrimental effect on dopaminergic transmission. To investigate this hypothesis, we use adeno-associated viral (AAV) vectors to overexpress human $\alpha$-syn in the rat substantia nigra. Moderate overexpression of either wild-type (WT) or A30P $\alpha$-syn differs in the motor phenotypes induced, with only the WT form generating hemiparkinsonian
\end{abstract}

Electronic supplementary material The online version of this article (doi:10.1007/s00401-012-0963-y) contains supplementary material, which is available to authorized users.

M. N. Gaugler · W. Bobela · S. Mohanna - J.-C. Bensadoun · P. Aebischer $(\square) \cdot$ B. L. Schneider $(\bowtie)$

Neurodegenerative Studies Laboratory, Brain Mind Institute, EPFL, AI 2241 Station 19, 1015 Lausanne, Switzerland e-mail: patrick.aebischer@epfl.ch

B. L. Schneider

e-mail: bernard.schneider@epfl.ch

O. Genc $\cdot$ R. Schneggenburger

Laboratory of Synaptic Mechanisms, Brain Mind Institute,

EPFL, Lausanne, Switzerland

M. T. Ardah · O. M. El-Agnaf

Department of Biochemistry, Faculty of Medicine and Health

Sciences, United Arab Emirates University, Al Ain, UAE

O. M. El-Agnaf

Faculty of Medicine, King Abdulaziz University,

Jeddah, Saudi Arabia

M. Cantoni - G. W. Knott

Centre of Interdisciplinary Electron Microscopy,

EPFL, Lausanne, Switzerland impairments. Wild-type $\alpha$-syn causes a reduction of dopamine release in the striatum that exceeds the loss of dopaminergic neurons, axonal fibers, and the reduction in total dopamine. At the ultrastructural level, the reduced dopamine release corresponds to a decreased density of dopaminergic vesicles and synaptic contacts in striatal terminals. Interestingly, the membrane-binding-deficient A30P mutant does neither notably reduce dopamine release nor it cause ultrastructural changes in dopaminergic axons, showing that $\alpha$-syn's membrane-binding properties are critically involved in the presynaptic defects. To further determine if the affinity of the protein for membranes determines the extent of motor defects, we compare three forms of $\alpha$-syn in conditions leading to pronounced degeneration. While membrane-binding $\alpha$-syns (wild-type and A53T) induce severe motor impairments, an N-terminal deleted form with attenuated affinity for membranes is inefficient in inducing motor defects. Overall, these results demonstrate that $\alpha$-syn overabundance is detrimental to dopamine neurotransmission at early stages of the degeneration of nigrostriatal dopaminergic axons.

Keywords $\alpha$-Synuclein - Neurotransmission . Dopamine $\cdot$ Substantia nigra $\cdot$ Motor behavior . Electron microscopy

\section{Introduction}

The protein $\alpha$-synuclein ( $\alpha$-syn) is mainly present in the presynaptic compartment and is considered to exert important functions in the control of neurotransmitter release. Mounting evidence indicates a protective role for $\alpha$-syn at the synapse, where synuclein proteins have a nonclassical chaperone activity facilitating the assembly of the 
SNARE complex $[6,10]$. Indeed, $\alpha$-syn has been suggested to participate in the regulation of neurotransmitter homeostasis and release in the adult brain, in particular in the dopaminergic neurons that are selectively vulnerable to Parkinson's disease (PD) [1, 2, 9, 34, 47, 57].

$\alpha$-Synuclein is also linked with pathogenic roles in the nervous system. Missense mutations in the $\alpha$-syn gene are a rare, but defined cause of PD [33, 43, 58] and the protein constitutes the major component of Lewy bodies [50], the histological hallmark of PD. Moreover, excess of the wildtype (WT) protein leads to PD in families with a multiplication of the $\alpha$-syn gene locus [11, 23, 49], and the difference in clinical severity between duplication and triplication families suggests a direct relation to gene dosage. Interestingly, polymorphisms in the $\alpha$-syn gene possibly implicated in the expression level of the protein have recently been linked with sporadic forms of the disease in genome-wide association studies [44, 48].

Therefore, it is conceivable that the intracellular accumulation of $\alpha$-syn can lead to functional perturbations in neurotransmission, as part of the disease process. Recent publications demonstrate that moderate WT $\alpha$-syn overexpression reduces the recycling vesicle pool at mouse glutamatergic synapses and inhibits exocytosis in dopamine neurons [39]. Functional deficits were further explored in hippocampal neuronal cultures derived from $\boldsymbol{\alpha}$ syn transgenic mice, revealing enlarged vesicles and evident deficits in neurotransmitter release [45]. At the nigrostriatal level, transgenic mice expressing a truncated form of $\alpha$-syn show an age-dependent dysfunctional redistribution of SNARE proteins in the striatum [18]. Defects in synaptic transmission and axonal transport were reported in rats injected with an AAV2 vector encoding $\alpha$-syn A53T [12]. However, it remains largely unknown what are the functional in vivo effects of overabundant $\alpha$ syn at nigrostriatal dopaminergic synapses and if impaired neurotransmitter release contributes to the parkinsonian motor phenotype. Here, we focus on the targeted overexpression of $\alpha$-syn in the nigrostriatal projections of the rat, a structure which displays selective vulnerability to $\alpha$-syn toxicity [21, 28, 35].

Our aim was to create a specific functional perturbation in dopamine transmission using a viral gene-delivery system that allows for the adjusted overexpression of $\alpha$ syn at levels causing only a minimal loss of dopaminergic neurons. We show that overabundant $\alpha$-syn diminishes striatal dopamine release in the remaining neurons and leads to impaired motor behavior. At the ultrastructural level, $\alpha$-syn causes a reduction in the density of presynaptic vesicles in the striatal neuropil, concomitant with indications of autophagic degeneration. By comparing the WT and A53T forms of $\alpha$-syn to lipid-binding deficient variants $[7,26,53]$, we demonstrate that these functional defects depend on the interaction of $\alpha$-syn with biological membranes.

\section{Materials and methods}

Plasmid construction

Human WT and A30P $\alpha$-syn cDNAs (GenBank accession no. NM_000345) were inserted into the pAAV-pgk-MCS backbone, modified from the serotype 2 pAAV-cmv-MCS (Stratagene, La Jolla, CA, USA) using standard cloning procedures. For the comparison between the WT, A53T and $\Delta 2-11$ forms of $\alpha$-syn, the cDNAs were inserted in the pAAV-pgk-MCS-WPRE backbone, containing the Woodchuck hepatitis virus post-transcriptional regulatory element (WPRE), using standard cloning procedures. The following primers were used to amplify the $\Delta 2-11$ sequence from the full-length $\alpha$-syn cDNA: $5^{\prime}$-ATGCATCGATA CCATGAAGGAGGGAGTTGTGGCTGCT- $3^{\prime}$ and $5^{\prime}$-AT GGCAGATCTTTAGGCTTCAGGTTCGTAGTCTTGA-3'.

Recombinant AAV2/6 production and titration

Recombinant pseudotyped rAAV2/6 were produced, purified and titrated as described before [14]. The titers measured for AAV-pgk- $\alpha$-syn-WT, AAV-pgk- $\alpha$-syn-A30P and AAV-pgk-GFP used were $8 \times 10^{10} \mathrm{TU} / \mathrm{ml}(4.1 \times$ $\left.10^{13} \mathrm{vg} / \mathrm{ml}\right), \quad 1 \times 10^{11} \mathrm{TU} / \mathrm{ml}\left(1.7 \times 10^{14} \mathrm{vg} / \mathrm{ml}\right)$ and $2.6 \times 10^{10} \mathrm{TU} / \mathrm{ml}\left(1.7 \times 10^{13} \mathrm{vg} / \mathrm{ml}\right)$, respectively. The vectors AAV-pgk- $\alpha$-syn $\Delta 2$-11-WPRE, AAV-pgk- $\alpha$-synWT-WPRE, and AAV-pgk- $\alpha$-syn A53T-WPRE had titers of $1.3 \times 10^{10} \mathrm{TU} / \mathrm{ml}\left(1.3 \times 10^{14} \mathrm{vg} / \mathrm{ml}\right), 1.3 \times 10^{10} \mathrm{TU} /$ $\mathrm{ml}\left(5.8 \times 10^{13} \mathrm{vg} / \mathrm{ml}\right)$ and $6.4 \times 10^{9} \mathrm{TU} / \mathrm{ml}\left(1.3 \times 10^{14}\right.$ $\mathrm{vg} / \mathrm{ml})$, respectively.

Stereotaxic unilateral injection into the SNpc of rats

Female adult Sprague-Dawley rats (Charles River Laboratories, France), weighing approximately $200 \mathrm{~g}$ were housed in a 12-h light/dark cycle, with ad libitum access to food and water, in accordance with Swiss legislation and the European Community Council directive (86/609/EEC) for the care and use of laboratory animals. Stereotaxic injections of AAV vectors were performed as described previously [14]. For complete lesion of the medial forebrain bundle (MFB), an adult female Sprague-Dawley rat received a unilateral injection of 6-hydroxydopamine (6-OHDA, Sigma, USA) at anterio-posterior $-2.6 \mathrm{~mm}$, medio-lateral $+1.7 \mathrm{~mm}$, dorso-ventral $-8.55 \mathrm{~mm}$, with an injection speed of $1 \mu \mathrm{l} / \mathrm{min}$. The concentration of 6-OHDA was at $20 \mu \mathrm{g}$ in $4 \mu \mathrm{l}$ of ascorbic-saline. 
HPLC analysis of dopamine and dopamine metabolites

Contents of dopamine and metabolites were measured as described before [14]. Results are expressed as a percentage of the non-injected hemisphere.

ELISA assays and western blot analysis for $\alpha$-syn

Brain samples of substantia nigra (SN) and striatum of both hemispheres were obtained from rats unilaterally injected in the SN with $\alpha$-syn vectors. These samples were homogenized in CelLytic buffer, consisting of mild detergent, bicine and 150 mM NaCl (Sigma-Aldrich Company Ltd., Dorset, England, Product Number: C3228), supplemented with a cocktail of protease inhibitors, including AEBSF, aprotinin, E-64, EDTA, and leupeptin (Calbiochem-Novabiochem Corporation, San Diego, CA), and centrifuged at $3,000 \times g$ for $30 \mathrm{~min}$. The supernatants were collected, total protein concentration adjusted to $0.5 \mathrm{mg} / \mathrm{ml}$, and then stored at $-80^{\circ} \mathrm{C}$.

Human $\alpha$-syn was measured using a sandwich ELISA as described previously, with modifications to improve the sensitivity for low quantities of $\alpha$-syn in biological samples [42]. A black 384-well ELISA plate (Nunc Maxisorb, NUNC, Denmark) was coated overnight at $4^{\circ} \mathrm{C}$ with $1 \mu \mathrm{g} / \mathrm{ml}$ of a monoclonal antibody recognizing residues 121-125 of human $\alpha$-syn (AB 211, Santa Cruz Biotechnology, USA) $(50 \mu \mathrm{l} / \mathrm{well})$ in $200 \mathrm{mM} \mathrm{NaHCO}_{3}$ at $\mathrm{pH}$ 9.6. For detection of total $\alpha$-syn, the plate was similarly coated with $1.25 \mu \mathrm{g} / \mathrm{ml}$ of the monoclonal anti- $\alpha$-syn antibody syn-1 (clone $42, \mathrm{BD}$ Transduction Laboratories, USA). The plate was washed four times with PBST (0.05\% Tween 20/PBS), and blocked for $2 \mathrm{~h}$ at $37^{\circ} \mathrm{C}$ with $100 \mu \mathrm{PBS}$ containing $2.5 \%$ gelatin (fish skin gelatin 45\%, Sigma, USA) and $0.05 \%$ Tween 20. After four washes with PBST, $50 \mu \mathrm{l}$ of brain lysate were added to each well and incubated for $2 \mathrm{~h}$ at $37^{\circ} \mathrm{C}$. Captured $\alpha$-syn was detected by $0.2 \mu \mathrm{g} / \mathrm{ml}$ of a polyclonal anti- $\alpha$-syn antibody raised against recombinant full-length human $\alpha$-syn (FL-140, Santa Cruz Biotechnology; $50 \mu \mathrm{l} /$ well), followed by $50 \mu \mathrm{l}$ of HRP-labeled goat anti-rabbit antibody in blocking buffer (1:5,000; Jackson ImmunoResearch Laboratories, West Grove, USA). Bound HRP activity was assayed by $50 \mu \mathrm{l}$ of enhanced chemiluminescent substrate (SuperSignal ELISA Femto Maximum Sensitivity Substrate, Pierce Biotechnology, Rockford, USA) per well, and relative light units were measured on a Victor ${ }^{3} 1420$ microplate reader (Wallac). A standard curve was established on wells of $50 \mu \mathrm{l}$ recombinant human $\alpha$-syn solution at different concentrations in blocking buffer. Recombinant human fulllength $\alpha$-syn was expressed in Escherichia coli and purified as described previously [25, 42]. Intra-assay and inter-assay coefficients of variation were $<10 \%$.

SDS-PAGE and immunoblotting: brain lysates were boiled in the sample loading buffer $(250 \mathrm{mM}$ Tris $\mathrm{HCl}$
pH 6.8, 10\% SDS, 30\% glycerol, 5\% $\beta$-mercaptoethanol, $0.02 \%$ bromophenol blue) for $10 \mathrm{~min}$. Samples $(25 \mu \mathrm{g} /$ lane) were then separated on $15 \%$ SDS-PAGE, 1 mm-thick gels. The separated proteins were transferred to nitrocellulose membranes $(0.45 \mu \mathrm{m}$; Whatman GmbH-Germany) at $90 \mathrm{~V}$ for $45 \mathrm{~min}$. The membranes were boiled for $5 \mathrm{~min}$ in PBS, and then blocked with $5 \%$ marvel dried skimmed milk/PBS-Tween-20 $0^{\mathrm{TM}}(0.05 \%$; PBST) for $1 \mathrm{~h}$. The membranes were probed overnight at $4{ }^{\circ} \mathrm{C}$ with the following primary antibodies: syn 211 mouse monoclonal antibody to human $\alpha$-syn (a.a. 121-125); or FL-140 rabbit polyclonal antibody, which recognizes human and rat a-syn (Santa Cruz Biotechnology). The membranes were washed several times with PBST, followed by incubation with HRP-conjugated goat anti-rabbit (Dako Ltd., Ely, UK), or goat anti-mouse (Dako Ltd.) as appropriate, for $60 \mathrm{~min}$. The protein bands were visualized by using ECL reagents (Pierce, Rockford, IL) as described by the manufacturer.

\section{Behavioral analysis}

Cylinder test, amphetamine-induced rotation (D-amphetamine sulphate: $2.5 \mathrm{mg} / \mathrm{kg}$ i.p., Sigma, St Louis, MO, USA), and apomorphine-induced rotation (apomorphine$\mathrm{HCl}: 0.1 \mathrm{mg} / \mathrm{kg}$ s.c., Sigma) were performed as described previously [14], before virus injection and at monthly intervals post-surgery. In the cylinder test, only the measurements with a minimum of 16 touches were included in the analysis.

\section{Immunohistological analyses and counting}

The rats were killed and tissue processed as described before [4]. For immunofluorescence, slices were washed in PBS, blocked in 10\% normal donkey serum (NDS) (Jackson ImmunoResearch, Suffolk, UK) and $0.1 \%$ Triton $\mathrm{X}-100$ in PBS for $2 \mathrm{~h}$ at room temperature, incubated overnight at $4^{\circ} \mathrm{C}$ with an anti- $\alpha$-syn antibody (sheep IgG, 1:800; AB5334P Chemicon) in blocking solution, and for $2 \mathrm{~h}$ at room temperature with a secondary antibody conjugated to a 488-Alexa Fluor dye (Molecular Probes, Invitrogen AG, Basel, Switzerland). They were washed and mounted on glass slides using MOWIOL medium.

Stereological estimates of Nissl-positive nigral neurons were obtained as described previously [15]. For brightfield microscopy analyses, the slices were treated, and the percentage of immunoreactive neurons and striatal dopaminergic innervation determined as described previously [4, 14]. Primary antibodies used were anti-tyrosine hydroxylase (TH) (rabbit IgG, 1:500; AB152 Chemicon) and anti-vesicular monoamine transporter 2 (VMAT2) (rabbit IgG, 1:2,000; AB1767 Chemicon). 


\section{Amperometry}

Amperometric measurements of dopamine release in striatal slices followed previously described methods [29, 37]. Coronal forebrain slices (200 $\mu \mathrm{m}$ thickness) on the level of the striatum were cut with a vibratome (Leica VT1000S, Leica Microsystems, Germany), using the rats 9 weeks after unilateral injection of either the WT, or A30P $\alpha$-syn construct. The coronal slices were severed at the midline and incubated for $1 \mathrm{~h}$ at $36^{\circ} \mathrm{C}$ in a solution composed of $125 \mathrm{mM} \mathrm{NaCl}, 25 \mathrm{mM} \mathrm{NaHCO} 3,2.5 \mathrm{mM}$ $\mathrm{KCl}, 1.25 \mathrm{mM} \mathrm{NaH} \mathrm{PO}_{4}, 1 \mathrm{mM} \mathrm{MgCl}, 2 \mathrm{mM} \mathrm{CaCl}$, and $25 \mathrm{mM}$ glucose at pH 7.4, bubbled with $95 \% \mathrm{O}_{2}$ and $5 \% \mathrm{CO}_{2}$. During recordings, the same solution at room temperature $\left(21-23^{\circ} \mathrm{C}\right)$ was used as extracellular solution.

For amperometry, we used commercially available carbon fiber electrodes of $5 \mu \mathrm{m}$ diameter (ALA Scientific Instruments, USA), insulated to the tip. On the day of recording, their tips were cut off to guarantee a fresh detection surface. We puff-applied a bath solution containing $10 \mathrm{mM}$ dopamine against the electrodes. Only electrodes which responded with currents of at least $500 \mathrm{pA}$ (usually, 1-2 nA) were used for subsequent amperometry. The carbon fiber was inserted into the dorsomedial striatum at the level of its largest area extent at a depth of $\sim 50 \mu \mathrm{m}$ from slice surface. To evoke dopamine release, the slices were perfused with a high $\mathrm{K}^{+}$solution, containing $70 \mathrm{mM} \mathrm{KCl}, 57.5 \mathrm{mM} \mathrm{NaCl}, 25 \mathrm{mM} \mathrm{NaHCO} 3,1.25 \mathrm{mM}$ $\mathrm{NaH}_{2} \mathrm{PO}_{4}, 1 \mathrm{mM} \mathrm{MgCl}, 2 \mathrm{mM} \mathrm{CaCl}$, and $25 \mathrm{mM}$ glucose. Current signals were recorded with a patch-clamp amplifier (EPC9/2; HEKA Elektronik, Lambrecht, Germany) at a detection potential of $+800 \mathrm{mV}$, and digitized at a sampling rate $20 \mathrm{kHz}$ using the Patchmaster software (HEKA). Measurements were performed in at least $n=4$ pairs of slices from each injected rat, using the non-injected and injected hemispheres selected at random. In this way, we obtained paired comparisons of amperometric current traces from the injected, and non-injected side for each rat. The experimenter was blinded to the injected construct. Current amplitudes were measured off-line using the IGOR Pro 6 software (Wavemetrics Inc., Portland, OR, USA).

\section{Electron microscopy}

Resin-embedded brain tissue was prepared for serial imaging in the focused ion beam scanning electron microscope using a protocol previously described [31], with the difference that deeply anesthetized rats were directly perfused with $2.5 \%$ glutaraldehyde and $2 \%$ paraformaldehyde in $0.1 \mathrm{M}$ phosphate buffer at $\mathrm{pH} 7.4$, and sections from fixed brains were vibratome sectioned at the level of the largest striatal surface, heavy-metal stained, dehydrated, and resin embedded.

From the resin-embedded sections, the region to be imaged was selected under a light microscope, mounted onto a blank resin block, and trimmed with an ultramicrotome, before being placed in a Zeiss NVision $40 \mathrm{FIB} /$ SEM microscope. The block face was imaged with an electron beam set at $1.4 \mathrm{keV}$. Pixel dwell time was $\sim 10 \mu$ s, and the total image size was $2,048 \times 1,536$ pixels. Block face milling was carried out with a gallium ion source of current of $30 \mathrm{pA}$ with a voltage of $30 \mathrm{keV}$. Milling depth was between 10 and $20 \mathrm{~nm}$, but measured after each imaging session using the cylindrical size of the mitochondria as a Ref. [16]. Image analysis was carried out using the Neurolucida confocal module for serial images (Microbrightfield, USA). In all image series the voxel size and contrast levels showed all membranous structures, and these were always visible in 3D. This ensured that synapses and vesicles could be counted as an absolute value within each volume without using any stereological estimation.

\section{Statistical analysis}

Data are expressed as average \pm standard error of the mean. Statistical analysis was performed using the Statistica software (StatSoft Inc., OK, USA). Level of significance was set at 0.05. Applied specific tests are indicated in the figure legends.

\section{Results}

Moderate nigrostriatal expression of human WT $\boldsymbol{\alpha}$-syn impairs motor behavior

For $\alpha$-syn expression, AAV vectors pseudotyped with a serotype 6 capsid were chosen because of their high tropism for nigral dopaminergic neurons [4, 14]. We injected each vector unilaterally into the $\mathrm{SN}$ of cohorts of adult Sprague-Dawley rats at a virus dose of $2.8 \times 10^{7}$ transducing units (TUs), which had previously been demonstrated to cause only minimal and non-specific loss of dopaminergic neurons in this brain area [14]. To determine whether WT $\alpha$-syn and its lipid-binding deficient A30P mutant are able to affect motor behavior in a regimen of mild unilateral $\alpha$-syn overexpression, we used several tests to detect possible motor asymmetries. When animals were injected with the WT form of $\alpha$-syn, we observed motor asymmetries in both spontaneous behavior and druginduced rotational behavior, which developed progressively over a period of 4 months following virus injection (Fig. 1). In the cylinder test, animals with nigrostriatal overexpression of WT $\alpha$-syn showed a significantly 

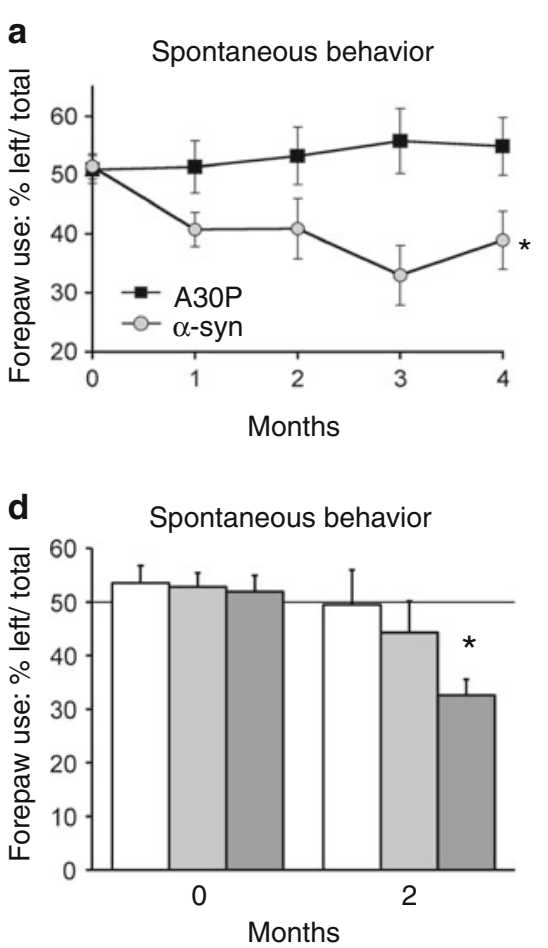

GFP $\square \alpha$-syn low dose

$\square \alpha$-syn high dose

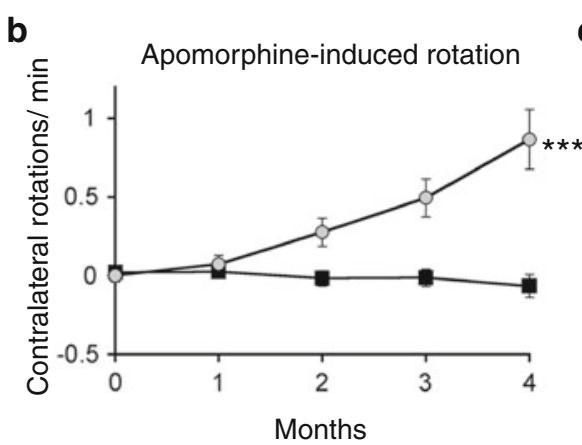

C
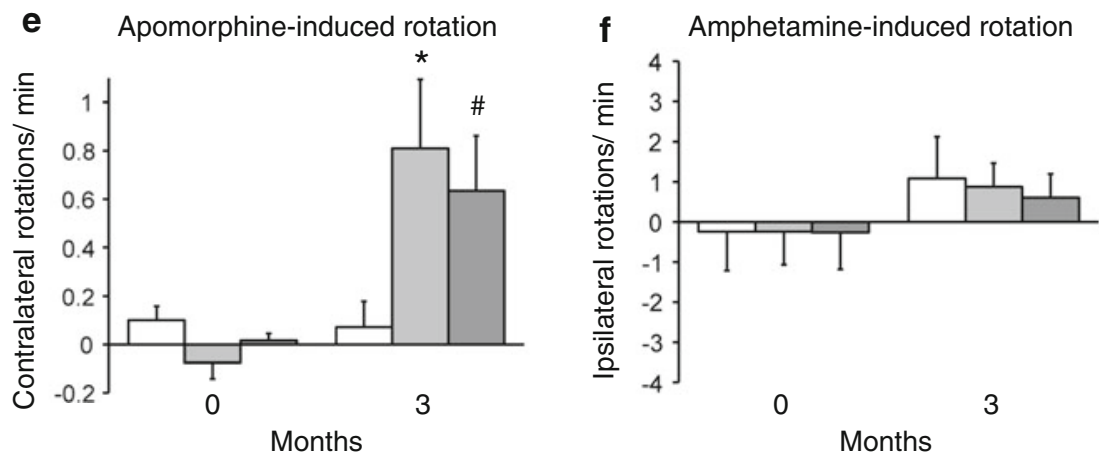

Fig. 1 WT $\alpha$-syn induces changes in spontaneous and drug-induced behavior over time. Motor asymmetries in rats injected with $2.8 \times 10^{7}$ TUs of AAV-pgk- $\alpha$-syn-WT $(n=18)$ and AAV-pgk$\alpha$-syn-A30P $(n=20)$ : a cylinder test, $\mathbf{b}$ apomorphine-induced rotametry, and $\mathbf{c}$ amphetamine-induced rotametry. Two-way repeated measure ANOVA with Newman-Keuls post hoc test: for (a) group effect, $F(1,36)=6.59, p<0.05$; for $(\mathbf{b})$ group $\times$ time, $F(4,144)=$ 14.62 , with $p<0.001$ at 5 weeks; for (c) group effect, $F(1,36)=$ $16.14, p<0.001$. Changes in spontaneous and drug-induced behavior depending on virus dose and comparison to a control GFP vector.

reduced use of the forepaw contralateral to the injected hemisphere, reaching $33 \pm 5 \%$ of total touches at 3 months post-injection (Fig. 1a). In the same animals, we observed the emergence of apomorphine-induced rotations (Fig. 1b), a behavioral effect that mainly corresponds to a postsynaptic sensitization of dopamine receptors. In contrast, animals overexpressing A30P $\alpha$-syn remained at baseline values for the entire length of the experiment in both tests (Fig. 1b, c). In amphetamine-induced motor behavior, neither WT nor A30P $\alpha$-syn caused a clear rotational phenotype (average rotation rates $\leq 1.5$ turns/ min; Fig. 1c). However, due to opposite trends of the rotations we noticed a significant difference between both groups, which might reflect subtle changes in dopamine distribution.

Comparing WT $\alpha$-syn and GFP vectors at the same dose of $2.8 \times 10^{7}$ TUs showed that the GFP vector did not cause any significant behavioral changes (Fig. 1d-f).
Rats for each condition were injected with either the AAV-pgk- $\alpha$-synWT at a dose of $1.4 \times 10^{7}(n=10)$ or $2.8 \times 10^{7}$ TUs of $(n=10)$, or with the AAV-pgk-GFP vector $(n=10)$ at a dose of $2.8 \times 10^{7}$ TUs. Motor asymmetry was measured using d the cylinder test, e apomorphine-induced rotametry, and $\mathbf{f}$ amphetamine-induced rotametry. Two-way repeated measure ANOVA with Newman-Keuls post hoc test for $(\mathbf{d})$ group $\times$ time, $F(2,25)=1.73,{ }^{*} p<0.05$ versus pre-test and GFP group; for (e) group $\times$ time, $F(2,27)=5.4$, with ${ }^{\#} p<0.05$ versus pre-test and $p=0.07$ versus GFP group and $* p<0.05$ versus pre-test and GFP group

In addition, we investigated whether the strength of the behavioral deficits depends on $\alpha$-syn expression level. The comparison between $1.4 \times 10^{7}$ TUs (low dose) and $2.8 \times 10^{7}$ TUs (high dose) of the WT- $\alpha$-syn coding vector showed a dose dependence of the effects of WT $\alpha$-syn on spontaneous behavior, with only the higher viral load displaying significant motor impairments (Fig. 1d). Animals overexpressing WT $\alpha$-syn also developed apomorphineinduced rotations, most apparent at 3 months (Fig. 1e). Again, we did not observe any significant rotational behavior in response to amphetamine (Fig. 1f). The dependence of behavioral impairments on vector dose is likely to reflect differences in the obtained striatal level of human $\alpha$-syn. Indeed, at 5 weeks post-injection, we compared the level of $\alpha$-syn in the striatum between the high $(n=4)$ and low vector $(n=5)$ doses using an ELISA assay for human $\alpha$-syn. The average human $\alpha$-syn level in the high vector dose group was increased on average 
$\sim 1.7$-fold when compared with the low dose, although this difference did not reach statistical significance (data not shown).

To explore the cause of the motor behavior, we analyzed histopathological alterations of dopaminergic markers in the nigrostriatal system.

WT $\alpha$-syn induces a moderate but more acute loss of dopaminergic markers than the A30P mutant

We next assessed the consequences of $\alpha$-syn overexpression on the loss of dopaminergic neurons in the $\mathrm{SN}$ pars compacta $(\mathrm{SNpc})$ and dopaminergic fiber density in the striatum.

Following injection of the $\alpha$-syn viral vectors, the recombinant human $\alpha$-syn protein was well detected in vivo by immunohistochemistry in the $\mathrm{SN}$ and the striatum (Fig. 2a, b). We also verified quantitatively that both forms of the protein presented a similar distribution and comparable protein levels between the SN and the striatum. In a separate cohort of animals, we measured expression levels 3 weeks after virus injection using an ELISA assay for human $\alpha$-syn. With an equivalent viral load $\left(1.4 \times 10^{7}\right.$ TUs), human $\alpha$-syn content was comparable between the WT and A30P constructs both in the SN $(170 \pm 49$ and $176 \pm 108 \mathrm{ng}$ human $\alpha$-syn/mg of total protein for WT $(n=5)$ and $\mathrm{A} 30 \mathrm{P}(n=5)$, respectively) and striatum (156 \pm 46 and $154 \pm 88 \mathrm{ng}$, respectively). This result was further confirmed by western blotting of striatal protein extracts revealing a specific $16 \mathrm{kDa}$ band in striatal protein extracts derived from the injected hemisphere with the human specific syn211 antibody (Fig. 2c). Using an ELISA assay detecting both rat and human $\alpha$-syn, we found a $2.5 \pm 0.4$ (WT, $n=5$ ) and $2.2 \pm 0.2(\mathrm{~A} 30 \mathrm{P}, n=5)$ fold increase in the total $\alpha$-syn level in the injected striatum, as compared with the non-injected hemisphere. There was no significant difference between both forms of $\alpha$-syn in the level of overexpression.

We assessed the effect of $\alpha$-syn expression on nigral neurons and striatal projections for the viral doses at which we observed differential motor disturbances (Fig. 2d-h). WT- $\alpha$-syn and A30P- $\alpha$-syn viruses were injected at either $1.4 \times 10^{7}$ (low dose) or $2.8 \times 10^{7}$ TUs (high dose).

At 3 months post-injection, overexpression of WT $\alpha$-syn led to a significant loss of neurons positive for VMAT2 in the SNpc (33 \pm 4.9 and $35 \pm 6.9 \%$, for respective doses) (Fig. 2d, e). Quantification of Nissl-positive neurons in the $\mathrm{SNpc}$ revealed a $26.5 \pm 4.6 \%$ reduction versus the noninjected side in the group injected with the highest dose of the WT- $\alpha$-syn coding vector, confirming that the neurons had indeed degenerated. In contrast, neuron numbers remained largely unchanged for A30P $\alpha$-syn $(4.0 \pm 3.1$ and $5.5 \pm 2.1 \%$ reduction for respective doses) (Fig. 2d, e). For comparison, an injected dose of $2.8 \times 10^{7}$ TUs of an AAV vector encoding GFP led to a loss of VMAT2positive neurons significantly lower than WT $\alpha$-syn, confirming the specific toxicity of human $\alpha$-syn (Fig. 2e).

We next examined the relative marker loss induced by the two forms of $\alpha$-syn in the SN's projection area. Measuring the density of VMAT2 immunoreactivity in the striatum, we found a decrease of $24.3 \pm 6.1 \%$ (low dose) and $25.1 \pm 4.9 \%$ (high dose) for WT $\alpha$-syn, and $15 \pm 2.6 \%$ (low dose) and $8.2 \pm 2.6 \%$ (high dose) for the A30P mutant (Fig. 2d, f). In addition, to confirm the $\alpha$-syninduced changes with a second dopaminergic marker, both analyses were repeated with $\mathrm{TH}$ immunohistochemistry (Fig. 2g, h). The measures obtained were comparable between VMAT2 and TH, except for the case of A30P, for which the VMAT2 density was slightly more reduced than TH density (Fig. 2f, h).

Overall, the histological analysis shows that the WT form of $\alpha$-syn causes significantly higher marker loss as compared to the A30P mutant. However, the overall level of degeneration even with WT $\alpha$-syn (25-35\%) does not provide a reasonable explanation for the observed functional impairment. Therefore, we set out to explore functional defects in the dopaminergic nigrostriatal projections that might explain the motor symptoms in the absence of overt degeneration of dopaminergic fibers.

Amperometric measurements reveal impaired evoked dopamine release following WT $\alpha$-syn overexpression

To explain the observed deficits in motor behavior, we next measured dopamine release in the striatum with carbon fiber amperometry [37, 54]. Since WT $\alpha$-syn, but not the A30P mutant induced motor deficits (Fig. 1), we compared the two forms of $\alpha$-syn, considering the A30P mutant as a control form of $\alpha$-syn with a loss of membrane-binding capacities [10, 39]. In order to minimize cell loss, all subsequent experiments were conducted with the lower dose of $\alpha$-syn-expressing vectors $\left(1.4 \times 10^{7} \mathrm{TUs}\right)$.

For animals overexpressing WT $\alpha$-syn, we found a significant reduction of amperometric signals in the injected relative to the non-injected hemisphere (Fig. 3). In the example of Fig. 3a, high $\mathrm{K}^{+}$caused an amperometric current response of $\sim 20 \mathrm{pA}$ in slices from the non-injected hemisphere (Fig. 3a, black traces), whereas the responses were reproducibly smaller in the striata of the injected hemisphere (Fig. 3a, red traces). In contrast, striata overexpressing A30P $\alpha$-syn had comparable amperometric responses for the non-injected, and the injected hemispheres (Fig. 3b, black and red traces, respectively). On average, amperometric responses were significantly reduced with WT $\alpha$-syn (from $36.4 \pm 6.4$ to $19.7 \pm 8.1$ $\mathrm{pA}$, or a reduction by $51.1 \% ; p<0.01$ ), whereas the relative reduction was not significant for the A30P form of 

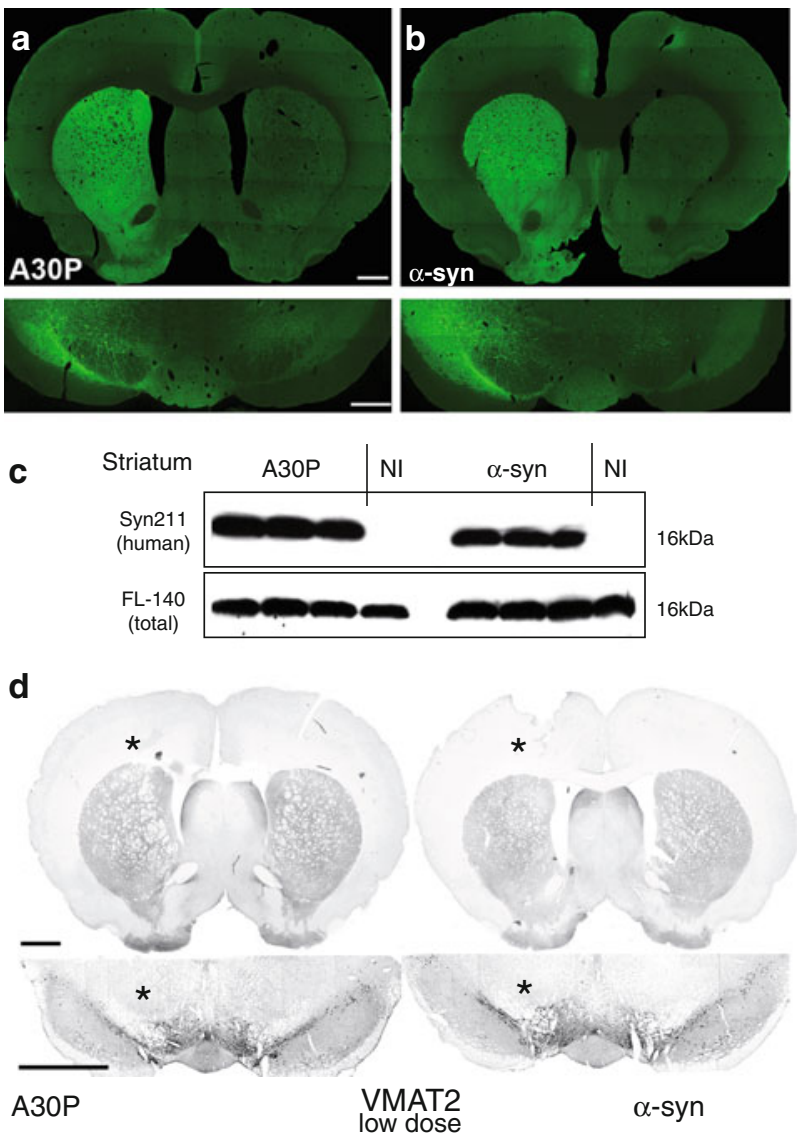

Fig. 2 WT $\alpha$-syn induces higher loss of dopaminergic markers than A30P $\alpha$-syn, while both forms of the protein show similar transgene expression in the $\mathrm{SN}$ and striatum. The animals were injected either with a high $\left(2.8 \times 10^{7} \mathrm{TUs}\right)$ or with a low dose $\left(1.4 \times 10^{7} \mathrm{TUs}\right)$ of vector. Expression of A30P (a) and wild-type $\alpha$-syn (b) in the SN (lower panel) and striatum (upper panel) of rats administered with high dose of an AAV vector as revealed by $\alpha$-syn immunostaining at 4 months post-injection. Scale bars: $1 \mathrm{~mm}$. c Western blot analysis of striatal proteins at 3 weeks post-injection (low dose), comparing three A30P-injected, three $\alpha$-syn WT-injected and two non-injected (NI) hemispheres. Antibodies were used to detect human (Syn211) or total $\alpha$-syn (FL-140). Note the modest increase in the level of total $\alpha$-syn in transduced striata. $\mathbf{d}$ Photomicrographs showing the loss of VMAT2-positive neurons and striatal fiber density of animals injected with low vector dose. The injected hemisphere is marked with an asterisk. e Loss of VMAT2-positive

$\alpha$-syn $(12.1 \pm 3.3 \%$; Fig. $3 \mathrm{c})$. These results demonstrate that mild overexpression of $\alpha$-syn causes a strong reduction of dopamine release that is specific to WT $\alpha$-syn and correlates with the observed deficit in spontaneous behavior.

Striatal content of dopamine and dopamine metabolites is reduced to a similar extent in WT and A30P $\alpha$-syn overexpressing animals

To determine whether reduced synthesis of dopamine or altered dopamine metabolism contributes to the observed dopamine release deficiency, we measured striatal levels of

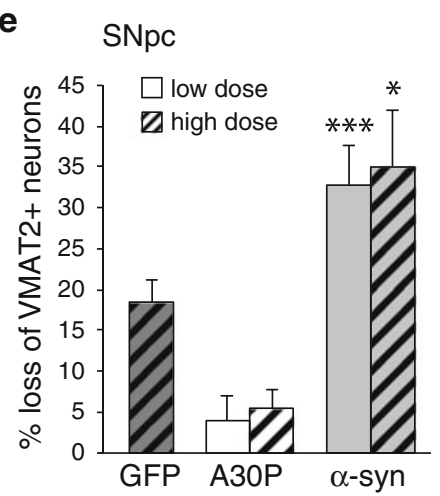

f Striatum

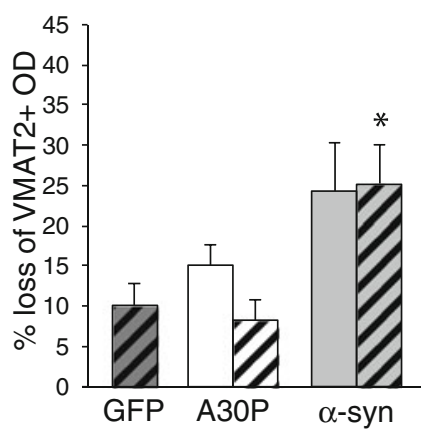

g

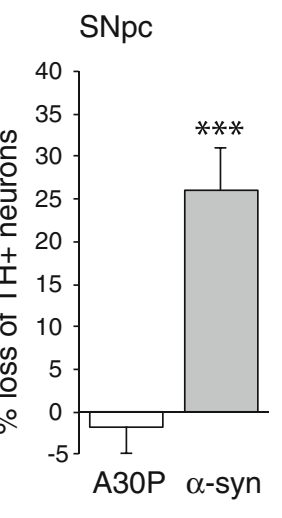

h

Striatum

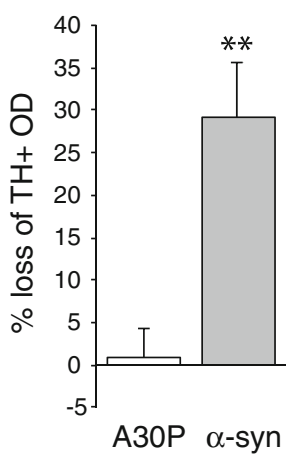

neurons in the SNpc of animals injected with AAV-pgk- $\alpha$-syn-A30P (low dose, $n=9$; high dose, $n=10$ ), AAV-pgk- $\alpha$-syn-WT (low and high dose, $n=10)$, and AAV-pgk-GFP $(n=10)$. f Decrease of VMAT2-positive striatal fiber density (A30P low and high dose: $n=8$ and 9; $\alpha$-syn-WT: $n=10$ for both conditions; GFP: $n=8$ ). Animals injected with the low dose of the $\alpha$-syn-WT or A30P vectors $(n=10$ in each group) were also analyzed for the following parameters: $\mathbf{g}$ loss of $\mathrm{TH}$-positive neurons in the $\mathrm{SNpc}, \mathbf{h}$ decrease of TH-positive striatal fiber density. All results are expressed as a percentage of the non-injected side. The histological analysis of the nigrostriatal system was performed 4 months post-vector injection. Comparisons were done by one-way ANOVA, followed by Newman-Keuls post hoc test $(\mathbf{e}, \mathbf{f})$ or two-tailed Student $t$-test $(\mathbf{e}-\mathbf{h}) ; * p<0.05$ versus the GFP and A30P groups (oneway ANOVA); $* * p<0.01$ and $* * * p<0.001$ versus the A30P group (Student $t$-test)

transmitter and metabolites in animals injected with $1.4 \times 10^{7}$ TUs of vector at 8 weeks post-injection. Overall, dopamine content was reduced in the injected hemisphere for both WT and A30P mutant $\alpha$-syn (to $77 \pm 9 \%$ and $81 \pm 2 \%$ of the non-injected hemisphere, respectively), while the decrease in levels of the metabolites DOPAC and HVA were overall less pronounced (Fig. 3d). The observed reduction in striatal dopamine levels was not specific to the WT protein and appeared to be in the same range as the observed dopaminergic marker loss for $\alpha$-syn WT (see above, Fig. $2 d-g$ ). We did not measure any significant change in the striatal concentration of serotonin (5-HT; Fig. 3d). 
a $\alpha$-syn
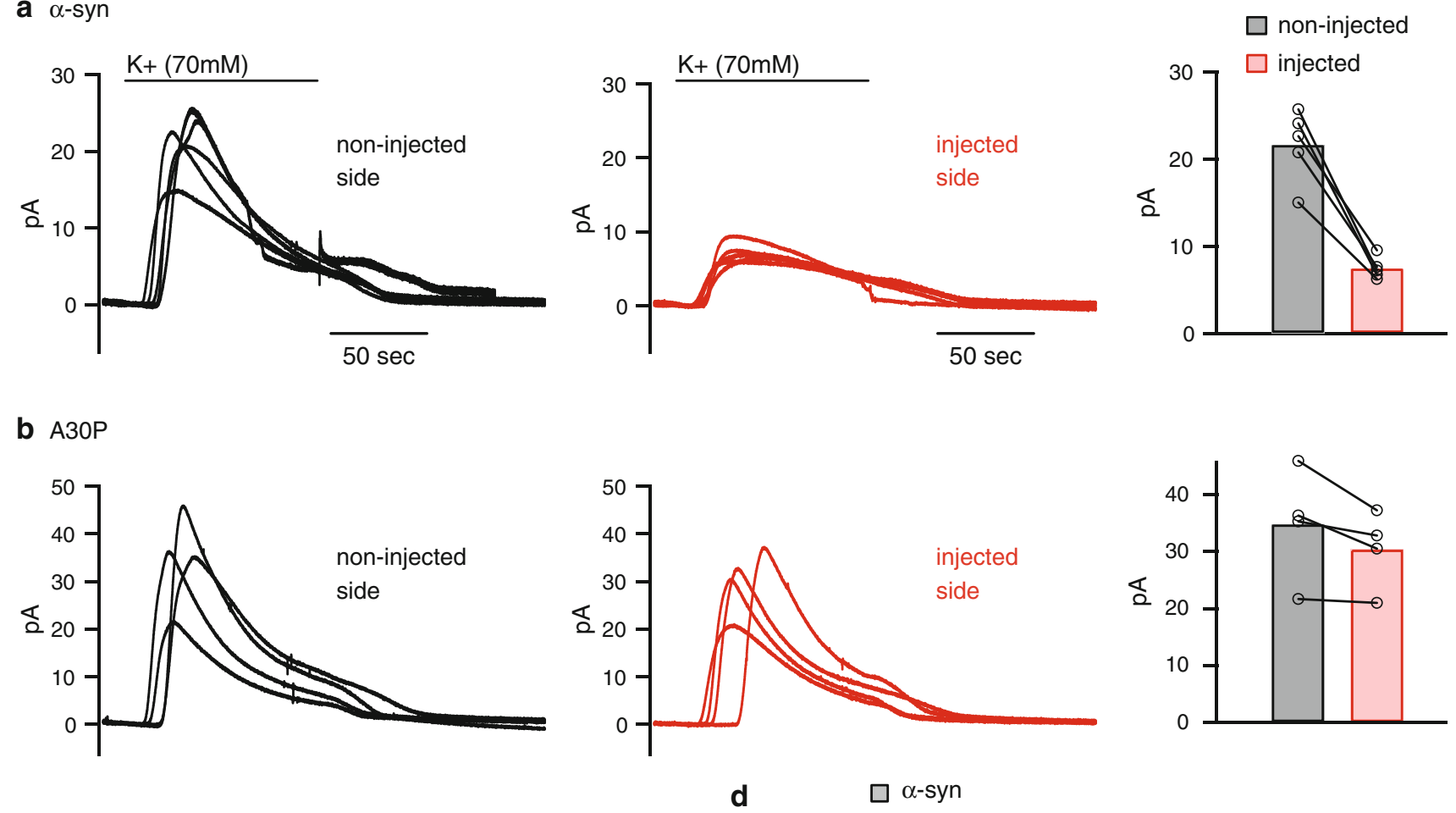

C non-injected side injected side
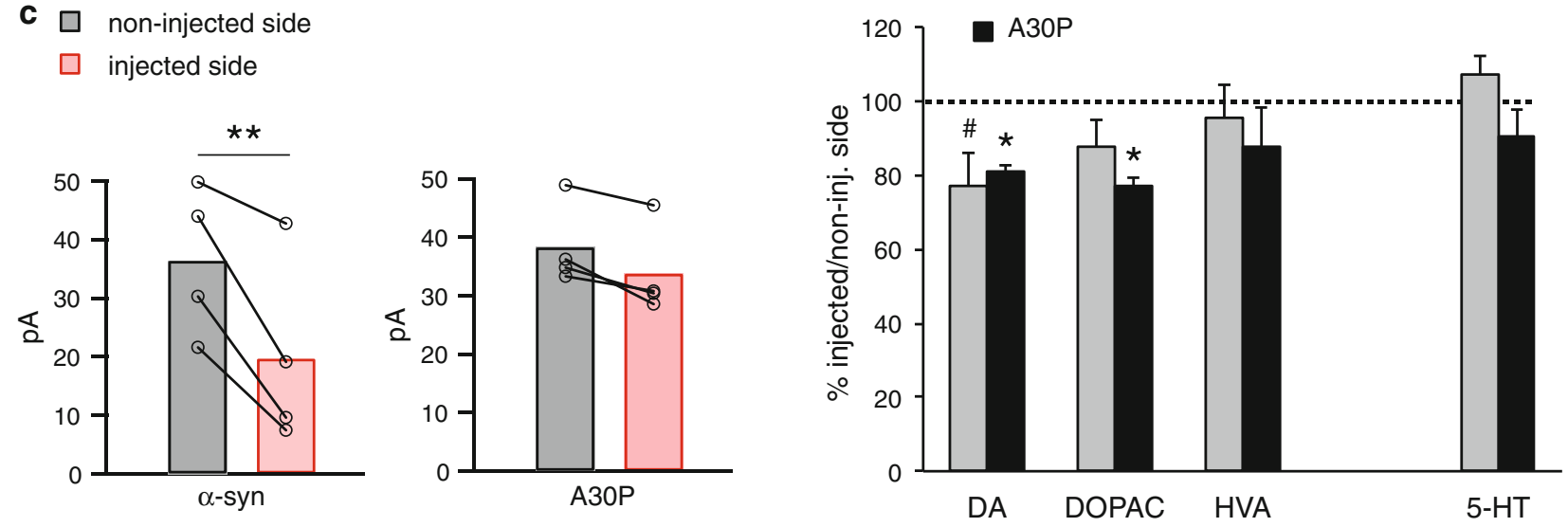

Fig. 3 Amperometric measurements show a reduction of evoked dopamine release in striata overexpressing WT $\alpha$-syn. a, b Representative traces of evoked current (with basal currents subtracted for individual curves) and plot of maximum amplitudes of all slice pairs for non-injected and injected hemispheres of individual rats administered with $1.4 \times 10^{7}$ TUs of AAV-pgk- $\alpha$-syn-WT (a) or AAV-pgk$\alpha$-syn-A30P (b). Across all measurements, the difference between injected and non-injected hemispheres was significantly different between WT- $\alpha$-syn $(n=19)$ and A30P- $\alpha$-syn $(n=17)$ overexpressing animals (two-way repeated measures ANOVA, followed by Tukey post hoc test, group effect: $p<0.05$ ). c Averages and distributions of maximum amplitudes of evoked current in the

Ultrastructural three-dimensional imaging of striatal neuropil and morphological identification of dopaminergic axons

In order to investigate possible presynaptic correlates of the reduced dopamine release, we studied the effect of $\alpha$-syn

injected and non-injected hemispheres of animals overexpressing WT $(n=4)$ or A30P $\alpha$-syn $(n=4) . * * p<0.01$ versus the non-injected hemisphere (two-way repeated measure ANOVA, followed by a Tukey post hoc test). d Striatal depletion of neurotransmitters and metabolites in the striatum. Total content of dopamine $(D A)$, DOPAC, HVA and 5-HT in the striata of animals killed at 8 weeks after injection with $1.4 \times 10^{7}$ TUs of virus (AAV-pgk- $\alpha$-syn-WT, $n=6$; AAV-pgk- $\alpha$-syn-A30P, $n=3$ ). Results are expressed as the percentage of remaining dopamine, DOPAC, HVA or 5-HT in the injected versus the non-injected hemisphere. ${ }^{*} p<0.05$ and ${ }^{\#} p=0.06$ (paired Student $t$-test)

on the ultrastructure of dopaminergic axons in the nigrostriatal system. We used a combined focused ion beam and scanning electron microscope (FIB/SEM) [31] to serially image at least two independent subvolumes in each condition, accounting for a total volume of $6,110 \mu \mathrm{m}^{3}$ of neuropil for WT $\alpha$-syn, $1,240 \mu \mathrm{m}^{3}$ for A30P $\alpha$-syn, and 
$2,280 \mu \mathrm{m}^{3}$ for non-injected control striatal tissue. Each series contained between 400 and 1,000 images with a spacing of $8-15 \mathrm{~nm}$ ( $\mathrm{z}$ spacing). With these datasets, dopaminergic axons could be identified and reconstructed in 3D.

Axonal projections within the striatal neuropil were classified by synapse type and vesicle morphology. Up to $92 \%$ of all synapses in the rat neostriatum are asymmetrical [24], corresponding for the greatest part to corticostriatal and thalamostriatal glutamatergic excitatory afferents, and to a smaller degree to a subgroup of striatal cholinergic collaterals. We identified glutamatergic synapses by asymmetrical contacts with prominently thickened postsynaptic varicosities (Fig. 4a, open arrowheads).

In some cases, glutamatergic synapses appeared to be in close proximity to synaptic contacts made on the same dendritic spines by a second class of axons with a distinct morphology (Fig. 4a, closed arrowhead). These synapses contained large, pleomorphic, mainly flattened vesicles with a lower contrast between vesicle membrane and lumen, as described before in immunolabeled dopaminergic terminals [17]. Vesicles were evenly distributed throughout the whole axonal length, with only small clusters at identifiable synapses (see below, Fig. 5a). These axons appeared highly homogenous and formed small en passant symmetrical synapses with dendritic spines and shafts (Fig. 4a, closed arrowheads), a previously reported ultrastructural feature of dopaminergic fibers [22]. Therefore, the morphology of this class of axons matched previously described attributes of dopaminergic terminals in the rat neostriatum.

We noticed two remaining axon subclasses that formed symmetrical synapses and were mainly classified according to the size and shape of their presynaptic vesicles. For each axon type, the diameters of individual vesicles were measured to illustrate differences in shape and size (Fig. 4b). GABAergic synapses were readily identifiable in our samples, with very broad synaptic contacts [5] and smallsized, round vesicles (Fig. 4b). Finally, a subpopulation of axons, referred to as type- $x$, was distinguished by relatively broad symmetrical synaptic contacts and large, mainly round vesicles (Fig. 4b) with a dense vesicle membrane
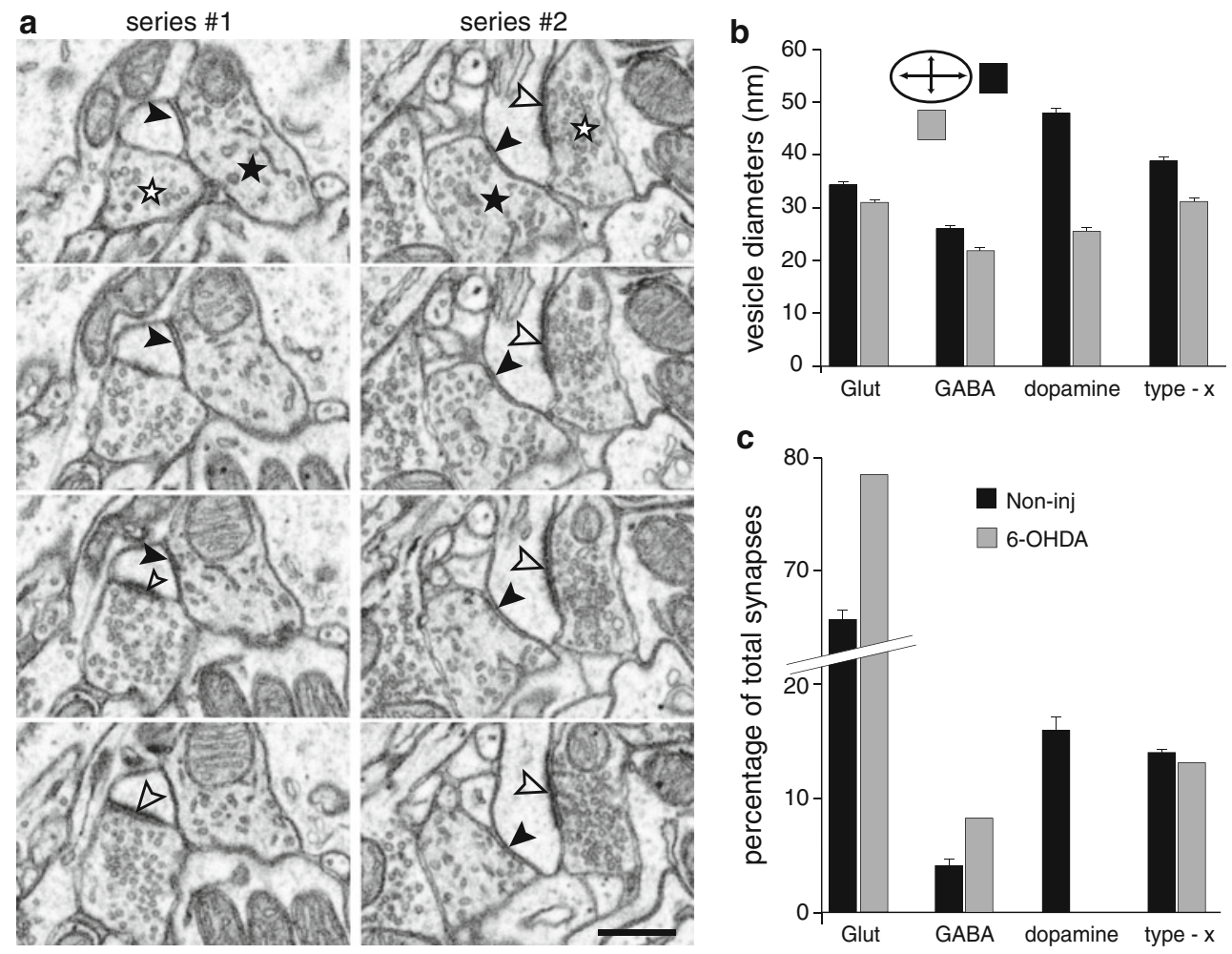

Fig. 4 Morphological identification of dopaminergic axons. a Two representative series of consecutive images showing a glutamatergic axon (open star) forming an asymmetric synaptic contact (open arrowhead) on a dendrite. This synapse is closely juxtaposed to a dopaminergic axon (closed star) forming a symmetric synaptic contact (closed arrowhead). Scale bar: $500 \mathrm{~nm}$. b For each type of axon identified in a non-injected rat striatum, we measured the small and large diameters of 50 individual vesicles present in axons and synaptic terminals. The diameters of vesicles were measured in the focal plane of the largest visible vesicle surface. Average diameter values are indicated for each axon type. Glut indicates glutamatergic vesicles. $\mathbf{c}$ Proportion of each synapse type, expressed as a percentage of total synapses in four control non-injected striatal samples (black bars). Note the complete elimination of synapses identified as dopaminergic in the striatum sub-volume of a 6-OHDA-lesioned animal (gray bars) 
Fig. 5 The overexpression of WT $\alpha$-syn induces a depletion of vesicles in dopaminergic axons. a Representative 3D reconstructions of dopaminergic axons in random volumes $(4 \times 4 \times 4 \mu \mathrm{m})$, with individual vesicles (red dots) and mitochondria, for a noninjected and a WT- $\alpha$-syn overexpressing hemisphere. b, c Vesicle density in dopaminergic axons from injected and non-injected hemispheres of WT- $\alpha$-syn overexpressing animals, normalized to either axon volume (b), or axon length (c). WT $\alpha$-syn: $\mathrm{n}=21$ injected, $n=20$ non-injected axons; A30P: $n=19$ injected, $n=24$ non-injected axons. Student $t$ test: $* * p<0.01$ a
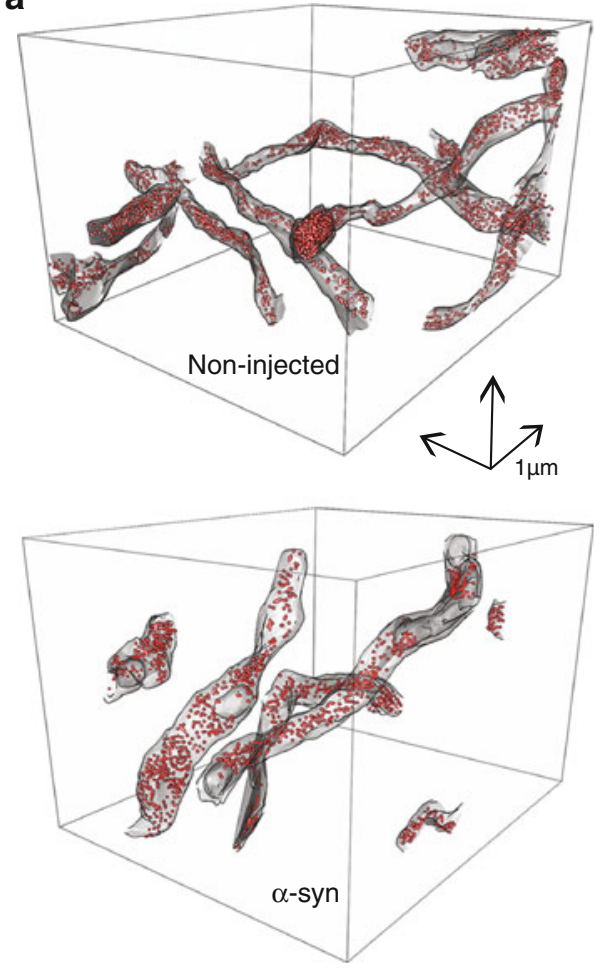

b

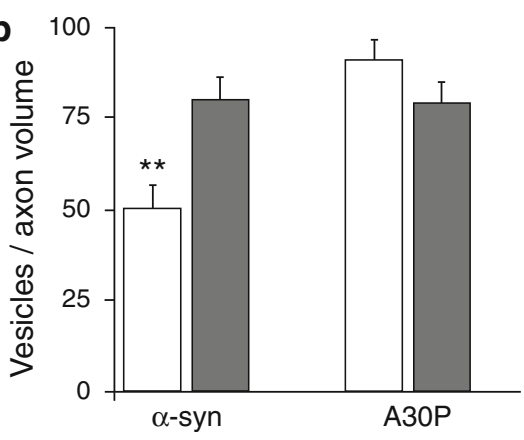

C

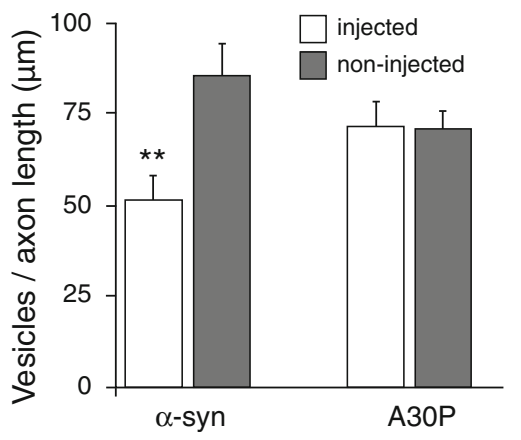

strongly contrasting an electron-lucent lumen (not shown). This class, as yet non-identified, appeared heterogeneous and might comprise multiple subtypes such as cholinergic or serotoninergic terminals. Supplementary movie 1 gives a representative sample of aligned FIB/SEM images of the rat striatal tissue, with identified synapse types.

To definitively demonstrate the nature of the dopaminergic axons identified by morphology, we repeated the same imaging procedure on the striatum of a rat whose medial forebrain bundle (MFB) had been lesioned with an injection of 6-OHDA, 3 weeks before analysis. Measuring the relative percentages of the four synapse types in the depleted striatum, we could no longer find the type of synapses we previously identified as dopaminergic ( $16 \pm 1.2 \%$ and $0 \%$, for control and 6-OHDA-lesioned striatum, respectively; Fig. 4c). Conversely, there was a relative increase in GABAergic and glutamatergic synapses in the 6-OHDA-lesioned striatum (Fig. 4c). Altogether, we conclude that morphological criteria allow for the identification of dopaminergic axons in FIB/ SEM images.

Quantitative ultrastructural analysis reveals a depletion of dopaminergic vesicles upon overexpression of WT $\alpha$-syn

Using the FIB/SEM analysis, we are now in a position to quantify ultrastructural changes in $3 \mathrm{D}$ reconstructed dopaminergic axons overexpressing human $\alpha$-syn. We analyzed striata of two rats per group, each injected with $1.4 \times 10^{7}$ TUs of WT- $\alpha$-syn or A30P- $\alpha$-syn virus at 10 weeks post-surgery. We determined vesicle density within 3D-reconstructed dopaminergic axons in striatal tissue (Fig. 5a). As we found dopaminergic vesicles widely distributed throughout neurites, we measured total numbers of axonal vesicles rather than vesicles situated around synaptic active zones.

Total vesicle counts were normalized either to axon volume (from which the volumes of mitochondria were subtracted) (Fig. 5b), or axon length (Fig. 5c). In animals with a nigrostriatal overexpression of WT $\alpha$-syn, the vesicle density was significantly reduced on the injected hemisphere. The number of vesicles in dopaminergic axons was diminished by $37 \pm 0.1 \%$ when normalized to axon length, and by $40 \pm 0.1 \%$ when normalized to axon volume, as compared to the vesicle density in the noninjected hemispheres (Fig. 5b, c). In contrast, A30P- $\alpha-$ syn did not reduce the density of dopaminergic vesicles (Fig. 5b, c). In addition, the size of dopaminergic vesicles was significantly increased in the hemisphere expressing WT $\alpha$-syn, as compared to the non-injected contralateral side. Measuring 50 vesicles across four axons per condition, the average large/small vesicle diameters were $67.4 \pm 0.4 / 40.7 \pm 0.3 \mathrm{~nm}$ in the WT- $\alpha$ syn hemispheres, as compared to $54.9 \pm 2.0 / 33.9 \pm$ $1.5 \mathrm{~nm}$ in the control non-injected side (Student $t$-test, $p<0.005)$. 
These results indicate a clear reduction in the density of vesicles in dopaminergic axons following overexpression of human $\alpha$-syn. The depletion of dopaminergic vesicles could underlie the impaired striatal dopamine release which we found in amperometric recordings.

WT $\alpha$-syn reduces the number of dopaminergic synapses and leads to the accumulation of autophagic structures in dopaminergic axons

In order to further assess possible ultrastructural changes that may explain the reduced dopamine neurotransmission, we quantified the abundance of synapses in dopaminergic axons. For each individual dopaminergic axon identified in random striatal subvolumes, we determined the number of synaptic contacts as defined by the presence of at least three vesicles clustered in close proximity to juxtaposed
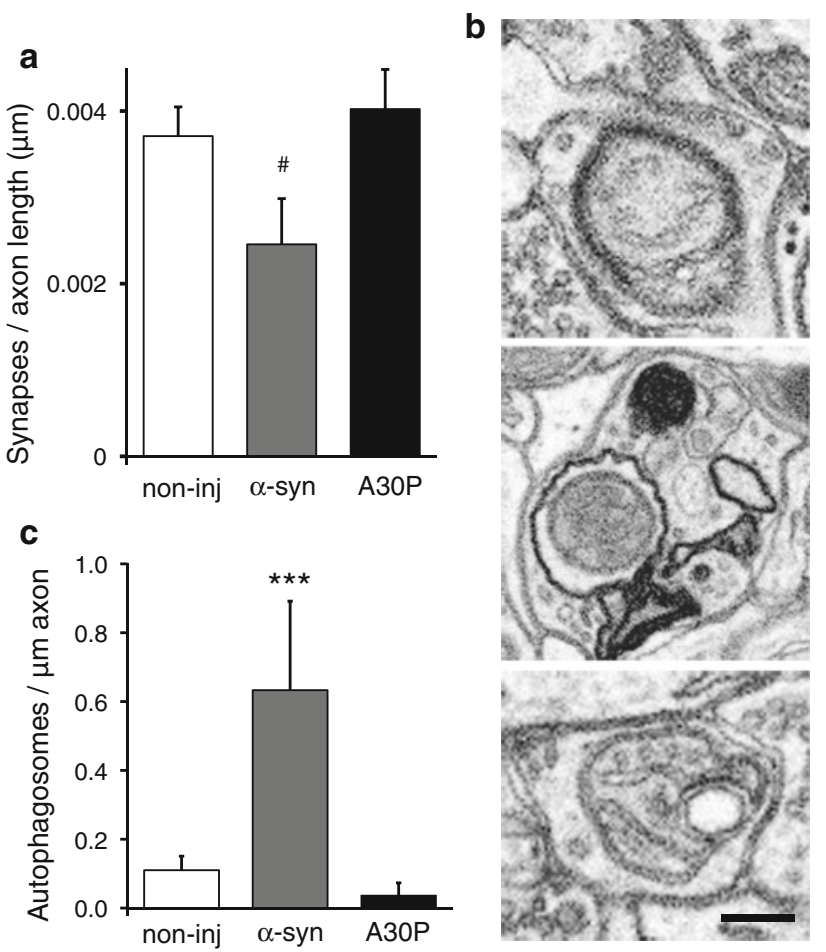

Fig. 6 The overexpression of WT $\alpha$-syn in dopaminergic axons induces a decrease in synapse numbers and an accumulation of autophagic structures. a Synapse density in dopaminergic axons from either non-injected $(n=42)$, WT $(n=20)$ or A30P $\alpha$-syn $(n=19)$ overexpressing hemispheres, normalized to axon length $(\mu \mathrm{m})$. Oneway ANOVA, $F(2,78)=3.0$, Newman-Keuls post hoc: ${ }^{\#} p=0.05$ versus non-injected control and $p<0.05$ versus A30P. b, c Autophagosomes accumulate in dopaminergic axons of animals expressing WT $\alpha$-syn. The density of autophagic structures per $\mu \mathrm{m}$ of axon (c) is significantly increased in response to WT $\alpha$-syn $(n=21)$, as compared to the non-injected controls $(n=47)$ and A30P mutant $(n=20)$. Scale bar: $250 \mathrm{~nm}$; Kruskal-Wallis test and bilateral comparison, $\mathrm{H}(2, n=88)=25.3: * * * p<0.01$ compared to the noninjected control group and $p<0.001$ compared to A30P mutant membranes (Fig. 6a). When compared to the non-injected hemispheres, we found a $34 \%$ reduction in the number of dopaminergic synapses, normalized to axon length, in striata injected with the WT- $\alpha$-syn virus. The A30P-mutant did not show any effect (Fig. 6a).

Ultrastructural examination of striatal tissue also revealed signs of distal degeneration confined to dopaminergic axons in hemispheres overexpressing WT $\alpha$-syn. Degenerating structures were composed of double- or triple-limiting membranes separated by an electron-lucent lumen or single-membrane wrapped mitochondria at early stages of demise (Fig. 6b). These features correspond to earlier descriptions of autophagosomes [13, 40]. The number of autophagic structures per axon length was determined in 84 individual dopaminergic axons (with an average length of $4.2 \mu \mathrm{m}$ ) from random striatal subvolumes of four non-injected, two WT- $\alpha$-syn and two A30P- $\alpha$-syn hemispheres, all at 10 weeks post-injection. Compared to both non-injected and A30P samples, we found approximately six times as many degenerating structures in animals overexpressing WT $\alpha$-syn (Fig. 6c).

These results further indicated ultrastructural changes in dopaminergic axons that are concomitant with early signs of neurodegeneration, which might contribute to the reduced dopamine release observed in amperometric recordings.

Correlation of impaired dopaminergic motor function with $\alpha$-syn association to biological membranes

We found above that the A30P $\alpha$-syn mutant, which is deficient for binding biological membranes [7, 26], does not cause motor impairments (Fig. 2) nor reduced dopamine release (Fig. 1), and fails to significantly reduce vesicle density in dopaminergic terminals (Fig. 5). We sought to determine if other forms of $\alpha$-syn would impact motor behavior as a function of their ability to bind membranes. Therefore, we compared WT $\alpha$-syn to the A53T mutant, which displays similar membrane-binding properties as the WT form. In parallel, we expressed $\alpha$-syn $\Delta 2-11$, a N-terminal deletion mutant in which the $\alpha$-helix formation critical for membrane association is destabilized [53]. To induce more pronounced degenerative effects, we used AAV vectors with a modified expression cassette designed for enhanced $\alpha$-syn expression (see "Materials and methods"). This expression cassette was shown by western blotting to express approximately 3.6-fold more human $\alpha$-syn than the previous vector following infection of HEK293T cells. In addition, we measured by ELISA a eight-fold increase on average in the level of human $\alpha$-syn in the rat SN as compared with an identical dose $\left(1.4 \times 10^{7} \mathrm{TUs}\right)$ of the previous vector, at 5 weeks postinjection (data not shown). 
We first infected HeLa cells with AAV vectors encoding $\alpha$-syn $\left(5 \times 10^{5}\right.$ TUs for $2.5 \times 10^{4}$ cells $)$ to assess the intracellular distribution of the WT and $\Delta 2-11$ forms of human $\alpha$-syn. Immunodetection of $\alpha$-syn (LB509 antibody) revealed a punctate staining for $\alpha$-syn, contrasting with the diffuse cellular distribution of the $\Delta 2-11$ mutant (data not shown), consistent with the previous observation that the deletion of the amino acids 2-11 affects the ability of human $\alpha$-syn to associate with biological membranes [53].

Next, we tested WT $\alpha$-syn, as well as the A53T and $\Delta 2$ 11 mutants for their ability to induce impairments in spontaneous motor behavior. The vectors were injected at a total dose of $1.25 \times 10^{7}$ TUs in the SNpc. The A53T mutant led to impaired spontaneous forepaw activity to an extent similar to WT $\alpha$-syn, reaching $20.8 \pm 3.3 \%$ and $24.6 \pm 2.1 \%$ of contralateral paw touches 4 months postinjection, respectively (Fig. 7a). Strikingly, $\alpha$-syn $\Delta 2-11$ failed to produce significant motor asymmetry, with $49.7 \pm 1.6 \%$ of contralateral forepaw activity. This result was further confirmed in apomorphine-induced rotational behavior. At 4 months, both the WT and A53T forms of $\alpha$-syn led to contraversive rotations (Fig. 7b), similar to the previous experiment (see Fig. 2). In contrast, $\alpha$-syn $\Delta 2-11$ did not produce significant rotational behavior up to 4 months post-injection. Amphetamine did not induce any significant rotational behavior with any of the three $\alpha$-syn forms (data not shown).

Histological analysis revealed a similar loss of nigral neurons as quantified by stereological counts of the dopaminergic marker TH (WT $\alpha$-syn: $60.3 \pm 4.5 \%$; A53T: $61.8 \pm 5.6 \%$; Fig. 7c, e). Interestingly, the extent of nigral neuron loss was significantly lower with the $\Delta 2-11$ variant $(26.6 \pm 6.2 \% ; p<0.001)$, although the protein also displayed some toxic effects in these conditions. In the striatum, the decrease in $\mathrm{TH}$ immunoreactivity reached $46.6 \pm 5.0 \%$ and $50.5 \pm 5.9 \%$ in the groups injected with the WT $\alpha$-syn and A53T vectors, respectively (Fig. 7d, f). Although $\alpha$-syn $\Delta 2-11$ caused, on average, a milder loss of TH-positive fibers $(28.6 \pm 8.6 \%)$, the difference did not reach significance $(p=0.07)$. Interestingly, immunostaining for human $\alpha$-syn (LB509 antibody) in the striatal tissue on the injected hemisphere revealed accumulations of WT $\alpha$-syn indicative of dystrophic fibers. In contrast, the $\Delta 2-11$ variant was homogenously distributed within axonal fibers with an overall normal morphology (Fig. $7 \mathrm{~g}$ ).

Altogether, these results confirm with two additional forms of the protein that the $\alpha$-syn variants able to associate with biological membranes (WT and A53T) are more prone to cause motor deficits. In contrast, the $\Delta 2-11 \alpha$-syn mutant with reduced affinity for membranes fails to impair forepaw motor activity, and produces less severe degenerative effects in nigral neurons.
Fig. 7 Using an AAV vectors for enhanced expression, both the WT and A53T forms of $\alpha$-syn lead to motor asymmetry, in contrast to the membrane-binding deficient $\Delta 2-11$ mutant. a Wild type and A53T $\alpha$-syn lead to spontaneous motor asymmetry as measured in the cylinder test, significantly different from the $\Delta 2-11 \alpha$-syn form. Twoway repeated measure ANOVA with Newman-Keuls post hoc test: group $\times$ time, $F(4,54)=10.25$, WT $\alpha$-syn: $n=16$, A53T: $n=7$, $\Delta 2-11: n=8 ; * p<0.05$ and $* * * p<0.001$ versus the other groups. b Wild type and A53T $\alpha$-syn progressively induce contraversive apomorphine-induced rotations, in contrast to the $\Delta 2-11 \alpha$-syn form. Two-way repeated measure ANOVA with Newman-Keuls post hoc test: group $\times$ time, $F(4,56)=2.58,{ }^{*} p<0.05$ versus A53T and $p=0.1$ versus $\alpha$-syn WT. c, d Representative photomicrographs of TH-immunostained nigral (c) and striatal sections (d) for each animal group. Asterisk indicates the injected hemisphere. e Loss of $\mathrm{TH}$-positive neurons in the SNpc and $\mathbf{f}$ loss of TH immunoreactivity (optical density) in the striatum as compared to the non-injected hemisphere. One-way ANOVA with Newman-Keuls post hoc: ${ }^{* * *} p<0.001$ and ${ }^{\#} p=0.07$ versus the other groups. $\mathrm{g}$ Photomicrographs of striatal tissue immunostained for human $\alpha$-syn. Note the presence of dystrophic axons only in the $\alpha$-syn WT animals (arrowheads). Scale bar: $20 \mu \mathrm{m}$

\section{Discussion}

Here, we report a motor behavioral phenotype specifically induced by the overexpression of $\alpha$-syn in rat nigral neurons. Unilateral expression of human $\alpha$-syn primarily leads to an asymmetry in spontaneous motor activity and to a mild rotational behavior in response to apomorphine. This motor phenotype is due to reduced dopamine release in the striatum, to an extent that clearly exceeds the measured loss of neurons, axons, and striatal dopamine. Seeking for ultrastructural correlates of this functional defect, we find a $40 \%$ loss of presynaptic vesicles in striatal dopaminergic terminals expressing $\alpha$-syn. Therefore, overabundance of $\alpha$-syn causes both a presynaptic deficit in vesicle genesis and/or recycling and early axonal degeneration that lead to reduced dopamine release and explain the detrimental effects on motor behavior.

In the experimental conditions employed here which lead only to mild degeneration, neuronal loss would account only for a $20-30 \%$ decrease in the striatal capacity for dopamine release, when compared to the intact hemisphere. Total dopamine content in the striatum is reduced to a similar extent. Although there is no significant decrease in dopamine metabolite content, we did not measure any clear change in the rate of dopamine metabolism, which would have been indicated by an increased DOPAC/dopamine ratio. Therefore, the lack of dopamine is primarily due to axonal fiber loss. This result contrasts to some extent with previous reports indicating increased dopamine metabolism induced by the A53T $\alpha$-syn mutant, when compared to a GFP-expressing control vector [12]. Experiments based on various degrees of intoxication with the selective toxin 6-OHDA indicate that a low level of 
a

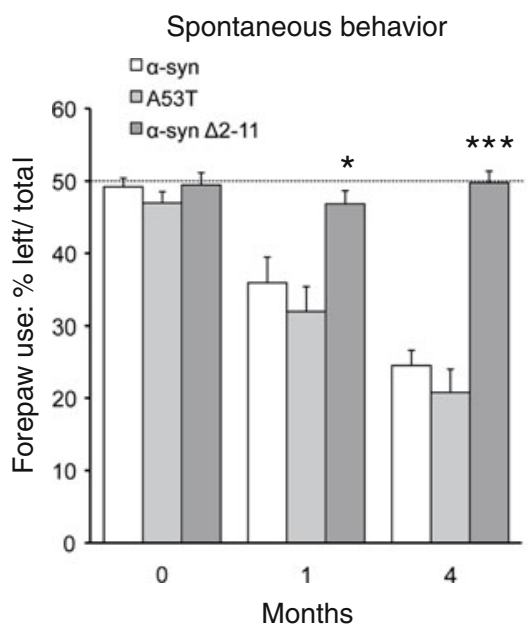

c

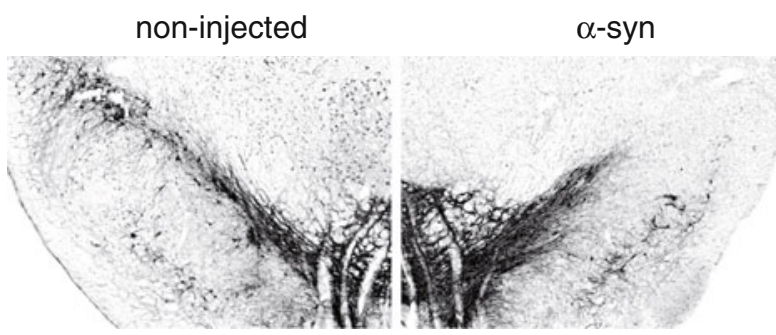

d

$\alpha$-syn

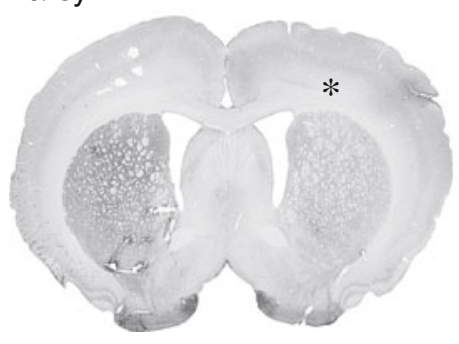

A53T

e
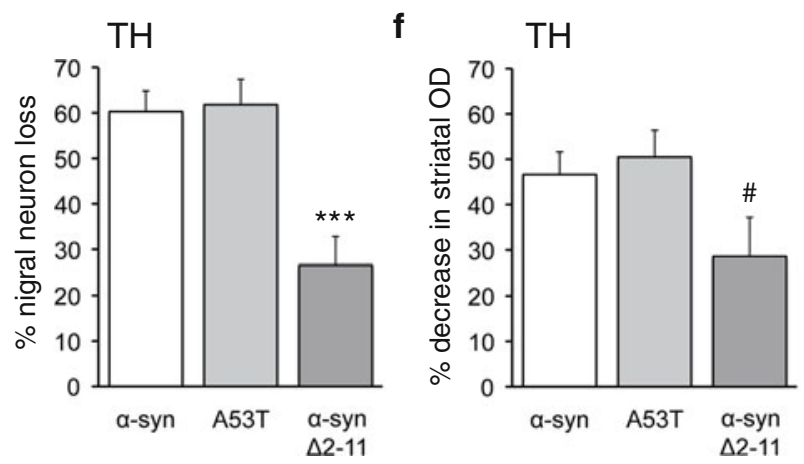

nigrostriatal damage, such as the one observed in our model system, is unlikely to produce measurable motor asymmetries [27]. Indeed, we do not observe any clear rotational behavior in response to amphetamine, the effect of which mainly relates to an asymmetric dopamine content in the striatum. Even more so, animals injected with the WT $\alpha$-syn vector tend to display contraversive
Apomorphine-induced rotation

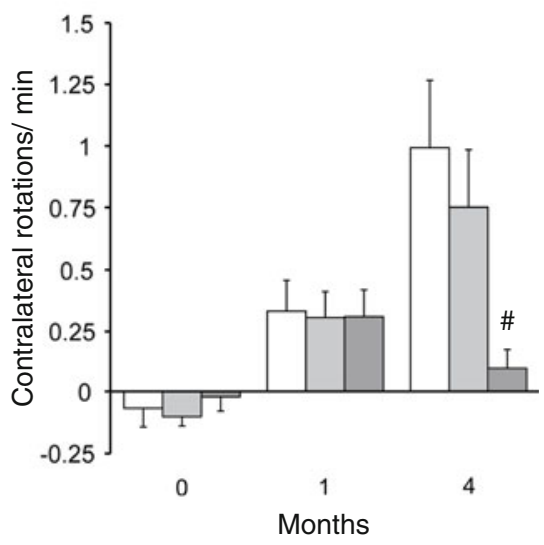

A53T

$\alpha-\operatorname{syn} \Delta 2-11$

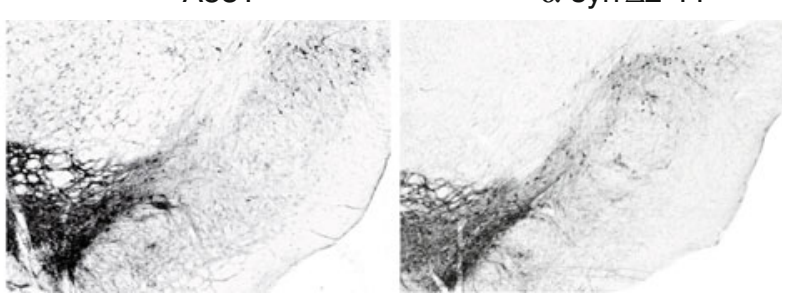

$\alpha-\operatorname{syn} \Delta 2-11$

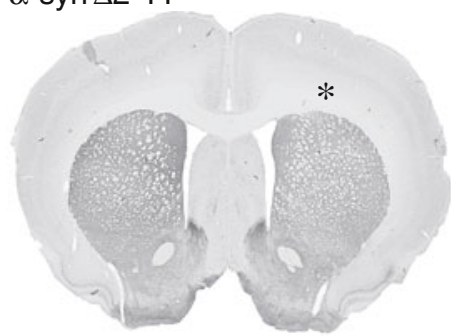

g

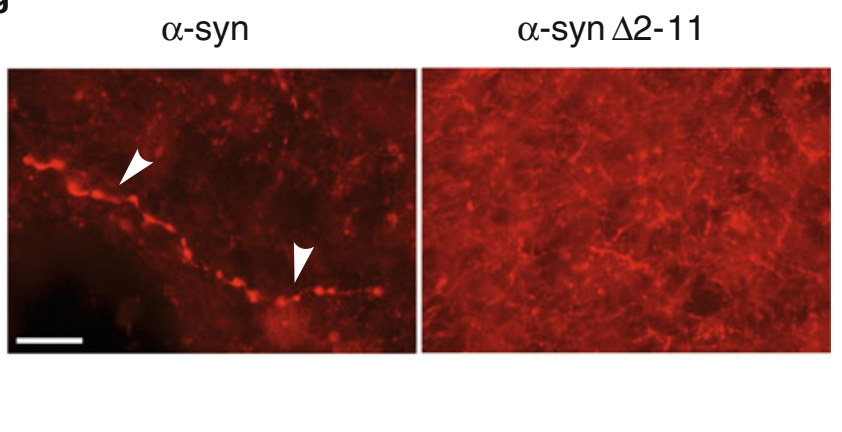

rotational behavior, suggesting that WT $\alpha$-syn may have subtle effects modulating amphetamine-induced dopamine release. Although such a contraversive rotational response to amphetamine has previously been documented and possibly associated with $\alpha$-syn-mediated inhibition of phospholipase D2 activity [20], further exploration will be needed to determine its exact cause. Nevertheless, 
amperometric recordings reveal an overall $\sim 50 \%$ reduction in depolarization-induced dopamine release following WT $\alpha$-syn overexpression. Therefore, it is safe to assume that spontaneous motor asymmetry in the cylinder test is largely caused by impaired dopamine release, which affects dopamine signaling despite only a minor loss of total striatal dopamine content. Similarly, the progressive development of apomorphine-induced rotations could reflect dopamine receptor hypersensitization in response to the reduced amount of dopamine release.

The clear phenotypic difference between the WT and A30P forms highlights the importance of functional deficits induced by $\alpha$-syn in the surviving nigral neurons. Indeed, despite a better preservation of the dopaminergic markers with the A30P mutant, both forms of $\alpha$-syn induce a similar loss in striatal dopamine content (Fig. 1d). The clear difference in motor behavior between the two forms of $\alpha$-syn therefore offers an opportunity to explore the cellular changes that closely correlate with this effect. These two forms of $\alpha$-syn differentially affect both depolarizationevoked dopamine release and presynaptic vesicle density. For the A30P mutant, these measures remain strikingly similar to the control non-injected side, highlighting the lack of effect on intracellular vesicle dynamics for this form of $\alpha$-syn.

The pathogenic A30P mutation reduces the affinity of $\alpha$-syn for biological membranes, similar to the rational $\Delta 2-11$ deletion which destabilizes $\mathrm{N}$-terminal $\alpha$-helix conformation [53]. Reduced affinity for membranes may impact on both the normal role and pathophysiological effects of the protein [3]. The absence of motor effects upon overexpression of the $\Delta 2-11$ and A30P mutants underlines the role that membrane association of $\alpha$-syn may have in dopamine neurotransmission. It is however possible that another biochemical property, such as the propensity to aggregate, further contributes to the observed differences between these forms of $\alpha$-syn. The lack of effect for the A30P mutant has been reported in several model systems. Notably, the A30P mutant fails to impair the growth of yeast cells [41], and does not produce the same effects as WT or A53T $\alpha$-syn in the maintenance and function of the neuronal presynaptic compartment [10, 39]. In the present study, we conclude that the overabundance of WT $\alpha$-syn, in contrast to the A30P and $\Delta 2-11$ mutants, interferes with the functioning of dopaminergic axons leading to reduced dopamine release and motor symptoms.

With respect to its pathogenic role, the A30P mutation may act in a different manner and lead to inheritable PD cases via the gain of an alternative toxic function. Previous reports found some negative effects of the A30P $\alpha$-syn mutant on dopamine release. In the mouse nigrostriatal system, A30P $\alpha$-syn was found to impact on vesicular release following high frequency stimulation to exhaust the readily releasable pool of striatal dopamine [56]. A second study using $\alpha$-syn-overexpressing chromaffin cells found a decrease in the releasable pool of catecholamine-containing vesicles [34]. Overall, although all forms of $\alpha$-syn may interfere at various levels with vesicular neurotransmitter release, our results suggest that membrane-binding forms correlate with more dramatic impairments of dopamine release in the rat nigrostriatal system.

In our experimental conditions, which intentionally lead to minor degeneration of the nigrostriatal system, the A30P mutant associated with an autosomal dominant form of PD is found to be less toxic than WT $\alpha$-syn. It is however clearly possible that the A30P mutant would display neuronal toxicity with higher vector dose and/or enhanced expression systems. Indeed, although the rare patients carrying the A30P mutation have a later age of disease onset as compared to patients with the A53T mutation or $\alpha$-syn triplication, they develop nigral degeneration $[32,46]$. While our results parallel some observations made in the yeast model, they contrast with the previously observed A30P toxicity obtained with nigral injections of lentiviral vectors $[35,55]$ and with one study using an AAV vector [30]. However, a recent study using an AAV2 vector similar to our model system also shows less toxicity with the A30P mutant than with WT $\alpha$-syn [52]. To our opinion, these discrepancies highlight the critical importance of parameters that include (1) the model organism, (2) the viral system employed to induce $\alpha$-syn overexpression, and (3) the resulting levels and kinetics of $\alpha$-syn overexpression. A30P $\alpha$-syn toxicity using AAV vector was also reported following a 1-year time course using a cba promoter that is likely to generate higher expression levels than the pgk-1 promoter used in the present study [30]. There was no direct comparison with WT $\alpha$-syn and no behavioral phenotype was observed despite a $>50 \%$ loss of nigral neurons. On the other hand, lentiviral and AAV preparations may differentially influence the context and conditions leading to $\alpha$-syn toxicity. Inherent vector properties determine the level of transgene expression and the time needed for the vector to achieve maximal expression. Although the pathologic response has been carefully addressed as a function of $\alpha$-syn levels in the yeast model [41], it is technically more difficult to precisely control $\alpha$-syn levels in nigral neurons using viral vector injections. We measured in brain tissue a two- to three-fold increase in levels of total $\alpha$-syn, consistent with previous studies using similar AAV vectors $[4,21]$. However, it is likely that the level of overexpression achieved in individual neurons is higher, considering the contribution of non-transduced cells in the protein extract. Furthermore, the preparations of AAV and lentiviral vectors notably differ in the degree of purification achieved. The potential presence of 
cellular contaminants in concentrated lentiviral suspensions may provoke local inflammatory reactions contributing to the induced phenotype. In conclusion, the various models currently available may reveal different aspects of the $\alpha$-syn pathology contributing to nigral degeneration and/or motor deficits.

While the A30P and $\Delta 2-11$ forms fail to impair motor behavior and tend to display less toxicity than WT human $\alpha$-syn in the nigrostriatal system, it remains unclear whether any mechanism may link these effects. In the yeast model, the impact of $\alpha$-syn on cellular functions relates to its expression level. While low levels of $\alpha$-syn primarily impair vesicular trafficking, higher levels lead to mitochondrial toxicity [51]. A similar gradation may exist in nigral neurons, with effects on vesicular dopamine release preceding overt degeneration. Therefore, it is possible that higher expression levels of the A30P mutant and/or prolonged expression will produce pathological effects similar to WT $\alpha$-syn in the AAV model system [30].

We observed a reduction of both vesicle density and the number of synapses in dopaminergic axons, providing a cellular correlate for the deregulation of nigrostriatal dopamine transmission upon WT $\alpha$-syn overexpression. A recent study explored the presynaptic effects of $\alpha$-syn overexpression using mouse glutamatergic synapses, reporting a reduced size of the recycling pool of vesicles and impaired clustering of vesicles after exocytosis [39]. While a smaller number of vesicles were found near the release zone, total vesicle numbers remained unchanged. In the nigral dopaminergic neurons which are primarily involved in PD motor symptoms, we observed a marked reduction in overall vesicle density, suggesting a deficiency in vesicle formation or turnover. Indeed, WT but not A30P $\alpha$-syn disrupts vesicle trafficking between the endoplasmic reticulum and the Golgi apparatus [19], which might contribute to a reduction in the pool of vesicles present at the level of the nerve terminals.

It will be important to determine in more details how the observed lack of presynaptic vesicles relates to dopamine release. Indeed, $\alpha$-syn specifically regulates either reserve or recycling pools of vesicles in glutamatergic synapses [8, 39]. It is unclear how this is linked to the overall reduction in vesicle density found in dopamine striatal axons. The lack of vesicles also suggests a possible re-distribution between vesicular and cytosolic dopamine [38]. It will be important to determine if $\alpha$-syn leads to elevated levels of dopamine in the cytosol in vivo. Indeed, cytosolic dopamine is prone to auto-oxidation, leading to oxidative stress [36] and possibly mitochondrial degeneration, which may contribute to $\alpha$-syn-induced neurodegeneration. Interestingly, we observed a drastic increase in autophagocytic structures often associated with degenerating mitochondria in nigrostriatal axons overexpressing WT $\alpha$-syn.
Overall, mild levels of human $\alpha$-syn in rat nigral neurons lead to a deficiency in dopamine release preceding outright neuron loss. In patients with $\alpha$-syn gene multiplications, as well as in sporadic forms of PD, the onset of motor symptoms may be due in part to functional perturbations that affect the release and storage of dopamine, and possibly contribute to the degeneration of the selectively vulnerable nigrostriatal system.

Acknowledgments The authors thank Vivianne Padrun, Fabienne Pidoux, Christel Sadeghi, Philippe Colin and Martial Mbefo Kamdem for their excellent technical assistance, and Gürdal Sahin and Prof. Deniz Kirik for the HPLC analysis. The authors are grateful to Alexandre Froidevaux for his help with the 3D-reconstruction from electron microscopic data. This work was supported by the MerckSerono EPFL Alliance, the Swiss National Science Foundation Grant No 31003A_120653, the European Community's FP7 under grant agreement no. HEALTH-F5-2008-222925 (Neugene), the Michael J Fox Foundation (Target Validation 2005), and a grant from the Synapsis Foundation (to R.S.).

\section{References}

1. Abeliovich A, Schmitz Y, Farinas I et al (2000) Mice lacking alpha-synuclein display functional deficits in the nigrostriatal dopamine system. Neuron 25:239-252

2. Anwar S, Peters O, Millership S et al (2011) Functional alterations to the nigrostriatal system in mice lacking all three members of the synuclein family. J Neurosci 31:7264-7274

3. Auluck PK, Caraveo G, Lindquist S (2010) alpha-Synuclein: membrane interactions and toxicity in Parkinson's disease. Annu Rev Cell Dev Biol 26:211-233

4. Azeredo da Silveira S, Schneider BL, Cifuentes-Diaz C et al (2009) Phosphorylation does not prompt, nor prevent, the formation of alpha-synuclein toxic species in a rat model of Parkinson's disease. Hum Mol Genet 18:872-887

5. Aznavour N, Mechawar N, Watkins KC, Descarries L (2003) Fine structural features of the acetylcholine innervation in the developing neostriatum of rat. J Comp Neurol 460:280-291

6. Burre J, Sharma M, Tsetsenis T, Buchman V, Etherton MR, Sudhof TC (2010) Alpha-synuclein promotes SNARE-complex assembly in vivo and in vitro. Science 329:1663-1667

7. Bussell R Jr, Eliezer D (2004) Effects of Parkinson's diseaselinked mutations on the structure of lipid-associated alpha-synuclein. Biochemistry 43:4810-4818

8. Cabin DE, Shimazu K, Murphy D et al (2002) Synaptic vesicle depletion correlates with attenuated synaptic responses to prolonged repetitive stimulation in mice lacking alpha-synuclein. J Neurosci 22:8797-8807

9. Chandra S, Fornai F, Kwon HB et al (2004) Double-knockout mice for alpha- and beta-synucleins: effect on synaptic functions. Proc Natl Acad Sci USA 101:14966-14971

10. Chandra S, Gallardo G, Fernandez-Chacon R, Schluter OM, Sudhof TC (2005) Alpha-synuclein cooperates with CSPalpha in preventing neurodegeneration. Cell 123:383-396

11. Chartier-Harlin MC, Kachergus J, Roumier C et al (2004) Alphasynuclein locus duplication as a cause of familial Parkinson's disease. Lancet 364:1167-1169

12. Chung CY, Koprich JB, Siddiqi H, Isacson O (2009) Dynamic changes in presynaptic and axonal transport proteins combined with striatal neuroinflammation precede dopaminergic neuronal 
loss in a rat model of AAV alpha-synucleinopathy. J Neurosci 29:3365-3373

13. Dunn WA Jr (1990) Studies on the mechanisms of autophagy: maturation of the autophagic vacuole. J Cell Biol 110:1935-1945

14. Dusonchet J, Bensadoun JC, Schneider BL, Aebischer P (2009) Targeted overexpression of the parkin substrate Pael-R in the nigrostriatal system of adult rats to model Parkinson's disease. Neurobiol Dis 35:32-41

15. Dusonchet J, Kochubey O, Stafa K et al (2011) A rat model of progressive nigral neurodegeneration induced by the Parkinson's disease-associated G2019S mutation in LRRK2. J Neurosci 31:907-912

16. Fiala JC, Harris KM (2001) Cylindrical diameters method for calibrating section thickness in serial electron microscopy. J Microsc 202:468-472

17. Freund TF, Powell JF, Smith AD (1984) Tyrosine hydroxylaseimmunoreactive boutons in synaptic contact with identified striatonigral neurons, with particular reference to dendritic spines. Neuroscience 13:1189-1215

18. Garcia-Reitbock P, Anichtchik O, Bellucci A et al (2010) SNARE protein redistribution and synaptic failure in a transgenic mouse model of Parkinson's disease. Brain 133:2032-2044

19. Gitler AD, Bevis BJ, Shorter J et al (2008) The Parkinson's disease protein alpha-synuclein disrupts cellular Rab homeostasis. Proc Natl Acad Sci USA 105:145-150

20. Gorbatyuk OS, Li S, Nha Nguyen F et al (2010) alpha-Synuclein expression in rat substantia nigra suppresses phospholipase D2 toxicity and nigral neurodegeneration. Mol Ther 18:1758-1768

21. Gorbatyuk OS, Li S, Sullivan LF et al (2008) The phosphorylation state of Ser-129 in human alpha-synuclein determines neurodegeneration in a rat model of Parkinson disease. Proc Natl Acad Sci USA 105:763-768

22. Groves PM, Linder JC, Young SJ (1994) 5-hydroxydopaminelabeled dopaminergic axons: three-dimensional reconstructions of axons, synapses and postsynaptic targets in rat neostriatum. Neuroscience 58:593-604

23. Ibanez P, Bonnet AM, Debarges B et al (2004) Causal relation between alpha-synuclein gene duplication and familial Parkinson's disease. Lancet 364:1169-1171

24. Ingham CA, Hood SH, Mijnster MJ, Baldock RA, Arbuthnott GW (1997) Plasticity of striatopallidal terminals following unilateral lesion of the dopaminergic nigrostriatal pathway: a morphological study. Exp Brain Res 116:39-49

25. Jakes R, Spillantini MG, Goedert M (1994) Identification of two distinct synucleins from human brain. FEBS Lett 345:27-32

26. Jensen PH, Nielsen MS, Jakes R, Dotti CG, Goedert M (1998) Binding of alpha-synuclein to brain vesicles is abolished by familial Parkinson's disease mutation. J Biol Chem 273: 26292-26294

27. Kirik D, Rosenblad C, Bjorklund A (1998) Characterization of behavioral and neurodegenerative changes following partial lesions of the nigrostriatal dopamine system induced by intrastriatal 6-hydroxydopamine in the rat. Exp Neurol 152:259277

28. Kirik D, Rosenblad C, Burger C et al (2002) Parkinson-like neurodegeneration induced by targeted overexpression of alphasynuclein in the nigrostriatal system. J Neurosci 22:2780-2791

29. Kitada T, Pisani A, Porter DR et al (2007) Impaired dopamine release and synaptic plasticity in the striatum of PINK1-deficient mice. Proc Natl Acad Sci USA 104:11441-11446

30. Klein RL, King MA, Hamby ME, Meyer EM (2002) Dopaminergic cell loss induced by human A30P alpha-synuclein gene transfer to the rat substantia nigra. Hum Gene Ther 13:605-612

31. Knott G, Marchman H, Wall D, Lich B (2008) Serial section scanning electron microscopy of adult brain tissue using focused ion beam milling. J Neurosci 28:2959-2964
32. Kruger R, Kuhn W, Leenders KL et al (2001) Familial parkinsonism with synuclein pathology: clinical and PET studies of A30P mutation carriers. Neurology 56:1355-1362

33. Kruger R, Kuhn W, Muller T et al (1998) Ala30Pro mutation in the gene encoding alpha-synuclein in Parkinson's disease. Nat Genet 18:106-108

34. Larsen KE, Schmitz Y, Troyer MD et al (2006) Alpha-synuclein overexpression in PC12 and chromaffin cells impairs catecholamine release by interfering with a late step in exocytosis. J Neurosci 26:11915-11922

35. Lo Bianco C, Ridet JL, Schneider BL, Deglon N, Aebischer P (2002) alpha-Synucleinopathy and selective dopaminergic neuron loss in a rat lentiviral-based model of Parkinson's disease. Proc Natl Acad Sci USA 99:10813-10818

36. Lotharius J, Brundin P (2002) Impaired dopamine storage resulting from alpha-synuclein mutations may contribute to the pathogenesis of Parkinson's disease. Hum Mol Genet 11:23952407

37. Mejias R, Villadiego J, Pintado CO et al (2006) Neuroprotection by transgenic expression of glucose-6-phosphate dehydrogenase in dopaminergic nigrostriatal neurons of mice. $\mathrm{J}$ Neurosci 26:4500-4508

38. Mosharov EV, Staal RG, Bove J et al (2006) Alpha-synuclein overexpression increases cytosolic catecholamine concentration. J Neurosci 26:9304-9311

39. Nemani V, Lu W, Berge V et al (2010) Increased expression of $\alpha$-synuclein reduces neurotransmitter release by inhibiting synaptic vesicle reclustering after exocytosis. Neuron 65:66-79

40. Nixon RA, Wegiel J, Kumar A et al (2005) Extensive involvement of autophagy in Alzheimer disease: an immuno-electron microscopy study. J Neuropathol Exp Neurol 64:113-122

41. Outeiro TF, Lindquist S (2003) Yeast cells provide insight into alpha-synuclein biology and pathobiology. Science 302:1772-1775

42. Paleologou KE, Kragh CL, Mann DM et al (2009) Detection of elevated levels of soluble alpha-synuclein oligomers in postmortem brain extracts from patients with dementia with Lewy bodies. Brain 132:1093-1101

43. Polymeropoulos MH, Lavedan C, Leroy E et al (1997) Mutation in the alpha-synuclein gene identified in families with Parkinson's disease. Science 276:2045-2047

44. Satake W, Nakabayashi Y, Mizuta I et al (2009) Genome-wide association study identifies common variants at four loci as genetic risk factors for Parkinson's disease. Nat Genet 41:13031307

45. Scott DA, Tabarean I, Tang Y, Cartier A, Masliah E, Roy S (2010) A pathologic cascade leading to synaptic dysfunction in alpha-synuclein-induced neurodegeneration. J Neurosci 30:80838095

46. Seidel K, Schols L, Nuber S et al (2010) First appraisal of brain pathology owing to A30P mutant alpha-synuclein. Ann Neurol 67:684-689

47. Senior SL, Ninkina N, Deacon R et al (2008) Increased striatal dopamine release and hyperdopaminergic-like behaviour in mice lacking both alpha-synuclein and gamma-synuclein. Eur J Neurosci 27:947-957

48. Simon-Sanchez J, Schulte C, Bras JM et al (2009) Genome-wide association study reveals genetic risk underlying Parkinson's disease. Nat Genet 41:1308-1312

49. Singleton AB, Farrer M, Johnson J et al (2003) alpha-Synuclein locus triplication causes Parkinson's disease. Science 302:841

50. Spillantini MG, Crowther RA, Jakes R, Hasegawa M, Goedert M (1998) alpha-Synuclein in filamentous inclusions of Lewy bodies from Parkinson's disease and dementia with lewy bodies. Proc Natl Acad Sci USA 95:6469-6473

51. Su LJ, Auluck PK, Outeiro TF et al (2010) Compounds from an unbiased chemical screen reverse both ER-to-Golgi trafficking 
defects and mitochondrial dysfunction in Parkinson's disease models. Dis Model Mech 3:194-208

52. Taschenberger $\mathrm{G}$, Garrido $\mathrm{M}$, Tereshchenko $\mathrm{Y}$, Bahr $\mathrm{M}$, Zweckstetter M, Kugler S (2012) Aggregation of alphaSynuclein promotes progressive in vivo neurotoxicity in adult rat dopaminergic neurons. Acta Neuropathol. doi:10.1007/s00401-011-0926-8

53. Vamvaca K, Volles MJ, Lansbury PT Jr (2009) The first N-terminal amino acids of alpha-synuclein are essential for alphahelical structure formation in vitro and membrane binding in yeast. J Mol Biol 389:413-424

54. Venton BJ, Troyer KP, Wightman RM (2002) Response times of carbon fiber microelectrodes to dynamic changes in catecholamine concentration. Anal Chem 74:539-546
55. Winner B, Jappelli R, Maji SK et al (2011) In vivo demonstration that alpha-synuclein oligomers are toxic. Proc Natl Acad Sci USA 108:4194-4199

56. Yavich L, Oksman M, Tanila $\mathrm{H}$ et al (2005) Locomotor activity and evoked dopamine release are reduced in mice overexpressing A30P-mutated human alpha-synuclein. Neurobiol Dis 20:303313

57. Yavich L, Tanila H, Vepsalainen S, Jakala P (2004) Role of alpha-synuclein in presynaptic dopamine recruitment. J Neurosci 24:11165-11170

58. Zarranz JJ, Alegre J, Gomez-Esteban JC et al (2004) The new mutation, E46 K, of alpha-synuclein causes Parkinson and Lewy body dementia. Ann Neurol 55:164-173 